\title{
Closed form fermionic expressions for the Macdonald index
}

\author{
Omar Foda ${ }^{a}$ and Rui-Dong Zhu ${ }^{b}$ \\ ${ }^{a}$ School of Mathematics and Statistics, \\ The University of Melbourne, Parkville, Victoria 3010, Australia \\ ${ }^{b}$ School of Theoretical Physics, Dublin Institute for Advanced Studies, \\ 10 Burlington Road, Dublin, Ireland \\ E-mail: omar.foda@unimelb.edu.au, nick_zrd@stp.dias.ie
}

ABSTRACT: We interpret aspects of the Schur indices, that were identified with characters of highest weight modules in Virasoro $\left(p, p^{\prime}\right)=(2,2 k+3)$ minimal models for $k=1,2, \ldots$, in terms of paths that first appeared in exact solutions in statistical mechanics. From that, we propose closed-form fermionic sum expressions, that is, $q, t$-series with manifestly non-negative coefficients, for two infinite-series of Macdonald indices of $\left(A_{1}, A_{2 k}\right)$ ArgyresDouglas theories that correspond to $t$-refinements of Virasoro $\left(p, p^{\prime}\right)=(2,2 k+3)$ minimal model characters, and two rank-2 Macdonald indices that correspond to $t$-refinements of $\mathcal{W}_{3}$ non-unitary minimal model characters. Our proposals match with computations from $4 \mathrm{~d} \mathcal{N}=2$ gauge theories via the TQFT picture, based on the work of J Song [75].

Keywords: Conformal and W Symmetry, String Duality, Supersymmetry and Duality, Conformal Field Theory

ARXIV EPRINT: 1912.01896

To Prof Barry M McCoy and "The Fermionic Characters" In memory of Professor Omar Foda 


\section{Contents}

\section{Introduction}

1.1 Schur and Macdonald indices in Argyres-Douglas theories as vacuum and $t$-refined vacuum $W_{N}$ characters 1

1.2 Schur indices in Argyres-Douglas theories in the presence of surface operators 2

1.3 Macdonald indices in Argyres-Douglas theories in the presence of surface operators

1.4 Virasoro characters as generating functions of weighted paths

1.5 Closed form expressions for the Macdonald index

1.6 Outline of contents and results

2 Definitions. The gauge theory side

2.1 The 3-parameter superconformal index of $4 \mathrm{~d} \mathcal{N}=2$ superconformal field theories

2.2 The Schur operators of $4 \mathrm{~d} \mathcal{N}=2$ superconformal field theories

2.3 The chiral algebra of $4 \mathrm{~d} \mathcal{N}=2$ superconformal field theories

2.4 The Schur index

2.5 Primary and descendant Schur and $W_{N}$ operators

2.6 The Macdonald index

2.7 Argyres-Douglas superconformal field theories

2.8 TQFT approach to Macdonald index

2.9 Song's work

3 Definitions. The statistical mechanics/combinatorics side

3.1 Alternating-sign (bosonic) sum expressions of the Virasoro characters

3.2 Constant-sign (fermionic) sum expressions of the Virasoro characters

3.3 Product expressions of the Virasoro characters

3.4 The work of Bressoud

3.5 The paths of Virasoro minimal model characters. The vacuum modules 3.5.1 The weight of a path

3.5.2 Higher- $k$ models 12

3.6 The paths of constant-sign Virasoro characters. The next-to-vacuum modules 15 3.6.1 Higher- $k$ models

3.7 A $T$-refinement of the constant-sign sum expressions of the Virasoro characters as Macdonald indices

4 A proposal for a closed-form expression for the Macdonald index

$\begin{array}{lll}\text { 4.1.1 Conjecture } 1 & 18\end{array}$

$\begin{array}{lll}\text { 4.1.2 Conjecture 2 } & 19\end{array}$

4.1.3 Conjecture 3: a path interpretation of aspects of the Schur index 19

$\begin{array}{ll}\text { 4.2 The Macdonald version of the sum expressions of the Virasoro characters } & 19\end{array}$ 
4.3 The sum expressions of the $\mathcal{W}_{3}$ characters 21

4.4 The Macdonald version of the sum expressions of the $\mathcal{W}_{3}$ characters 21

4.5 The ASW sum expressions of $\mathcal{W}_{3}$ characters 23

4.6 Matching the Virasoro infinite-series of vacuum characters 24

4.7 Matching the Virasoro infinite-series of next-to-vacuum characters 24

4.8 Matching the $\mathcal{W}_{3}$ vacuum and next-to-vacuum characters 25

4.9 Relation with Schur operators 25

5 Comments $\quad 26$

$\begin{array}{lll}5.1 & \text { Surface operators and characters } & 26\end{array}$

$\begin{array}{ll}5.2 & \text { Refining the bosonic version of a character }\end{array}$

5.3 The works of Bourdier, Drukker and Felix 27

5.4 The works of Beem, Bonetti, Meneghelli, Peelaers and Rastelli 27

5.5 Paths, particles, instantons, BPS states and the Bethe/Gauge correspondence 27

5.6 The thermodynamic Bethe Ansatz 28

My great collaborator, Prof. Omar Foda, passed away shortly after the completion of the third version of this paper. In fact, he shared the core idea of this paper with me back in 2017. It took me rather a long period to prepare and develop the tools we needed in this article, but I still feel lucky enough to finish this work with Omar. He first mentioned his illness to me in last September, when we started to summarize our results into an article. However, he was never willing to tell me too much about his health condition, and after certain times of surgeries, I thought he completely recovered from his illness, as he was so positive during our discussion on this paper. We even discussed about a lot of potential future directions after this work in December, and Omar also played the major role in the discussion with other research groups and the revision of this paper after we put it on the arXiv. I never thought he would leave us so soon.

Prof. Omar Foda was one of the most important people for me during my early academic life. He was also a kind and active collaborator for me. His brilliant ideas will continue to guide us in the future. May he rest in peace.

\section{Introduction}

\subsection{Schur and Macdonald indices in Argyres-Douglas theories as vacuum and $t$-refined vacuum $W_{N}$ characters}

In $[10,11,28]$, Beem et al. showed that the Schur indices in certain Argyres-Douglas theories are characters of irreducible highest-weight vacuum modules in a class of nonunitary $W_{N}$ minimal models. In [75], Song proposed a method to compute the Macdonald indices that generalizes the Schur indices of $[10,11]$ as $q$, $t$-series expansions of $t$-refined irreducible highest-weight vacuum modules in the non-unitary Virasoro minimal models $\mathcal{M}^{2,2 k+3}, k=1,2, \cdots$. 


\subsection{Schur indices in Argyres-Douglas theories in the presence of surface op- erators}

In [66], Nishinaka et al. studied the Schur indices in Argyres-Douglas theories in the presence of surface operators. They considered two infinite series of Argyres-Douglas theories, (1) the series labeled $\left(A_{n-1}, A_{m-1}\right)$ with $\operatorname{gcd}(n, m)=1$, and (2) the series labeled $\left(A_{n-1}, A_{2 m}\right)$, for $n=2, m=1,2, \cdots$, in the presence of the surface operator labeled by $s_{i}$, $i=1, \cdots, n-1$. They showed that in these two infinite series, the Schur index matches the character of the $\mathcal{W}$-algebra highest weight module with the same label $s_{i}, i=1, \cdots, n-1$. This generalizes the work of $[23-28,75]$ on the vacuum modules, and the work of [29-31] on the non-vacuum modules, which also involves surface operators in gauge theory. ${ }^{1}$ In the present work, we focus on the first series whose dual is the $W_{N}$ minimal model labeled by $\left(p=n, p^{\prime}=n+m\right)^{2}$

\subsection{Macdonald indices in Argyres-Douglas theories in the presence of surface operators}

In [85], Watanabe et al. extended the results of [66] to the corresponding Macdonald indices. Sum expressions for the Macdonald indices were obtained in terms of Macdonald polynomials for the series $\left(A_{n-1}, A_{m-1}\right), \operatorname{gcd}(n, m)=1$, for $n=2,3$, as a generalization of the results of [76]. For $n=2$, Macdonald indices could be computed to arbitrary high orders, but for $n=3$, the Macdonald index was determined from this approach only to a high order $\left(\mathcal{O}\left(q^{10}\right)\right)$. Due to the technical complication in the Higgsing method used in [85] to generate surface operators in Argyres-Douglas theories, only two infinite series of rank-2 Macdonald indices, the series that corresponds to the vacuum modules, and the series that corresponds to the next-to-vacuum modules of $\mathcal{W}_{3}$ characters, were conjectured.

\subsection{Virasoro characters as generating functions of weighted paths}

The local height probabilities in restricted solid-on-solid models (which are off-critical 1point functions on the plane with specific boundary conditions) are generating functions of weighted paths $[5,50]$. They are also equal to the characters of Virasoro minimal models (which are critical partition functions on the cylinder with specific boundary conditions). ${ }^{3}$ hence the latter have the same combinatorial interpretation as weighted paths. There is more than one way to represent these weighted paths, and in this work, we adopt the representation of these weighted paths proposed in [48].

The generating functions of these weighted paths admit more than one $q$-series representation. One of these representations is a constant-sign sum with manifestly non-negative coefficients. The coefficient $a_{n}$ of $q^{n}$ in this representation is the multiplicity of the states of conformal dimension $n$ (up a possible shift common to all states) in the corresponding irreducible highest-weight module. In $[14,16,17,47,57,81-83]$ these states were interpreted

\footnotetext{
${ }^{1}$ See $[12,13,19,35-37,41,67,68,77-80]$ for recent progress.

${ }^{2}$ In minimal models, usually the modules are labeled by positive integers $r_{i}$ and $s_{i}, i=1, \cdots, n$. However, due to the constraints $\sum_{i=1}^{n} r_{i}=p$ and $\sum_{i=1}^{n} s_{i}=p^{\prime}$, for $p=n$, only $s_{i}$ for $i=1, \cdots, n-1$ are free parameters left.

${ }^{3}$ The literature on this equivalence is extensive. For a comprehensive overview, and discussion motivation, we refer the reader to [43].
} 
in terms of (quasi-)particles and their weights (the corresponding power of $q$ ) were interpreted in terms of their (quasi-)momenta. These manifestly non-negative sum expressions were called 'fermionic characters',, 5

\subsection{Closed form expressions for the Macdonald index}

In [42], and independently [69], it was noted that Song's $q, t$-series for the vacuum modules of $\mathcal{M}^{2,2 k+3}, k=1,2, \cdots$ are generated by a specific $t$-refinement of the fermionic form of the corresponding Virasoro characters. In the present work, we extend and check this observation.

We show that (1) aspects of the $\mathcal{W}_{2}$ Schur index can be read directly from the paths, including the multiplicities of the Schur operators that contribute to the index, the composition of these operators in terms of Schur operators that are not derivatives of simpler ones (we call these 'primary Schur operators'as defined in 2.5) and Schur operators that are derivatives of simpler ones (we call these 'descendant Schur operators'as defined in 2.5), as well as the precise counting of the derivatives, and (2) that a refinement of these sum expressions in terms of a parameter $t$ with a specific power that depends on the numbers of particles, gives a closed form expression for the corresponding Macdonald character. We match our results with direct computations from the Argyres-Douglas theory side, based on a method proposed by J Song [75] and find complete agreement in cases where results are available from both sides.

\subsection{Outline of contents and results}

In section 2 and 3 , we introduce basic definitions that we need in the sequel, from the gauge theory side and from the statistical mechanics side, respectively, including the superconformal index, the Schur operators, the fermionic forms of the characters of the Virasoro $\left(p, p^{\prime}\right)=(2,2 k+3)$ non-unitary minimal models $(k=1,2, \ldots)$, as well a specific $\mathcal{W}_{3}$ nonunitary minimal model. Based on the fermionic form of the characters, we review the quasi-particle picture of the Virasoro minimal models, and define natural $t$-refined characters for these models by assigning different $t$-weights to different particle species. In section 4 , we conjecture that the $t$-refined character is equal to the Macdonald index computed from the gauge theory side, based on the observation that they match as series expansions in $q$, up to a high order. Next, we make the stronger conjecture that the quasi-particles of statistical mechanics are in one-to-one correspondence with the Schur operators that are counted by the Schur/Macdonald index in the gauge theory. Section 5 contains a number of comments.

Remark. We focus on the Virasoro characters, two infinite series of which are considered in this work. Following that, we discuss the case of two $\mathcal{W}_{3}$ characters separately and in analogous terms.

Remark. While we normally use the terminology t-refinements to add a parameter $t$, it is often convenient to think in terms of $T$-refinements instead where $T:=t / q$.

\footnotetext{
${ }^{4}$ The papers $[81,82]$ focus on the unitary minimal models, using the combinatorics of the paths that are appropriate to the unitary models, while [47] completes the proof in this case.

${ }^{5}$ There is another approach to the fermionic characters using path algebras of fusion graphs [56, 58, 59].
} 


\section{Definitions. The gauge theory side}

We recall basic definitions from the gauge theory side.

\subsection{The 3-parameter superconformal index of $4 \mathrm{~d} \mathcal{N}=2$ superconformal field theories}

The superconformal index is defined $[4,60]$ as the 3 -parameter Witten index

$$
\mathcal{I}(p, q, t)=\operatorname{tr}\left((-1)^{F} p^{\frac{E-2 j_{1}-2 R-r}{2}} q^{\frac{E+2 j_{1}-2 R-r}{2}} t^{R+r} e^{-\beta H}\right)
$$

where $\left(E, j_{1}, j_{2}, R, r\right)$ are the quantum numbers associated to the $\mathcal{N}=2$ superconformal algebra, that is, the dilatation charge, the spins, $\mathrm{SU}(2)_{R}$ charge and $\mathrm{U}(1)_{r}$ charge, $F$ is the fermion number and the Hamiltonian $H$ can be chosen ss $^{6}$

$$
H=2\left\{\overline{\mathcal{Q}}_{1 \dot{-}}, \overline{\mathcal{Q}}_{1 \dot{-}}^{\dagger}\right\}=2\left(E-2 j_{2}-2 R+r\right)
$$

The local operators that contribute to the superconformal index are BPS operators annihilated by $H$, or equivalently by $\overline{\mathcal{Q}}_{1-}$.

\subsection{The Schur operators of $4 \mathrm{~d} \mathcal{N}=2$ superconformal field theories}

The superconformal index depends on three fugacity parameters, $p, q$ and $t$. One can consider some special limit of the index, where the Hilbert subspace contributing to the index is further restricted. The Macdonald limit, $p \rightarrow 0$, restricts the index to local operators that are not only annihilated by the Hamiltonian, but also satisfy

$$
E-2 j_{1}-2 R-r=0,
$$

or equivalently

$$
E=j_{1}+j_{2}+2 R, \quad r+j_{1}-j_{2}=0
$$

These are called Schur operators. We refer the readers to [10] for the conventions and discussions used here, with a (limited) list of possible Schur operators.

\subsection{The chiral algebra of $4 \mathrm{~d} \mathcal{N}=2$ superconformal field theories}

In [10], a systematic method was discovered to construct a chiral algebra spanned by the Schur operators of $4 \mathrm{~d} \mathcal{N}=2$ superconformal field theories. The dual chiral algebra contains the Virasoro algebra with central charge $c_{2 d}$ given by the $c$-coefficient, $c_{4 d}$, in the 4 -point function of stress tensors in $4 \mathrm{~d}$, as

$$
c_{2 d}=-12 c_{4 d}
$$

\footnotetext{
${ }^{6}$ A review of the $4 \mathrm{~d} \mathcal{N}=2$ superconformal algebra can be for example found in $[10] .\{\bullet, \bullet\}$ denotes the anti-commutator of fermionic operators.
} 


\subsection{The Schur index}

The Schur index is the Schur limit, $p \rightarrow 0, q=t$, of the superconformal index and coincides with the character of the vacuum irreducible highest weight module of the corresponding chiral algebra

$$
\mathcal{I}(q)=\operatorname{tr}\left((-1)^{F} q^{h}\right),
$$

where the conformal weight of a $2 \mathrm{~d}$ chiral algebra state is

$$
h=R+j_{1}+j_{2},
$$

in $4 \mathrm{~d}$ terms.

\subsection{Primary and descendant Schur and $W_{N}$ operators}

In this work, we study isomorphisms between the Schur sector in 4d superconformal field theories and irreducible highest weight modules in $2 \mathrm{~d}$ chiral algebras.

Elements in the Schur sector (the set of all Schur operators) can be classified (as we show in the sequel) into a set of finitely-many Schur operators that are not descendants of other Schur operators under the action of the $4 \mathrm{~d}$ superconformal algebra, ${ }^{7}$ and a set of Schur operators that are descendants of other Schur operators under the action of the $4 \mathrm{~d}$ superconformal algebra (that is, the action with derivatives on the first set of Schur operators). In the sequel, we call the first type primary Schur operators, and the second type descendant Schur operators. ${ }^{8}$

An irreducible highest weight module in a $2 \mathrm{~d} W_{N}$ minimal conformal field theory consists (as well known) of a single highest weight state created by the action of a primary $W_{n}$ operator on the vacuum state (in the case of the vacuum highest weight module, the primary $W_{n}$ operator is the identity), and infinitely many descendant states that are generated by the action of $W_{N}$ operators on the highest weight state. We call the first type primary $W_{N}$ operators, and the second type descendant $W_{N}$ operators. ${ }^{9}$.

Each Schur operator in a Schur sector of the type studied in this work is in bijection with a $W_{N}$ operator. However, since there are (as we will show) in general finitely-many primary Schur operators in a Schur sector and one primary $W_{N}$ operator in a $W_{N}$ irreducible highest weight module, only one primary Schur operator maps to that primary $W_{n}$ operator, while the remaining primary Schur operators map to $W_{n}$ descendant operators. In the sequel, it is convenient to restrict the definition of primary Schur operators to those that map to descendant $W_{N}$ operators (in other words, we exclude the Schur operator that maps to the primary $W_{N}$ operator).

As we show in the sequel, one of the results of this work is that the primary Schur operators (that map to descendant $W_{N}$ operators) are distinguished in the sense that they create the particles that make the spectrum of the $2 \mathrm{~d} W_{N}$ minimal conformal field theory.

\footnotetext{
${ }^{7}$ The property that there are finitely-many such operators may be true only in the Argyres-Douglas theories/minimal models studied in this work. It is possible that the Schur sectors of more general models have infinitely many primary Schur operators.

${ }^{8}$ Note that this terminology is new, we introduce it for the purposes of this work.

${ }^{9}$ These are the known primary and descendant $W_{N}$ generators. We use $W_{n}$ when necessary to avoid confusion.
} 
A known example of a primary Schur operator which maps to a $W_{n}$ descendant operator is the R-symmetry current in the stress tensor multiplet, which maps to a $W_{n}$ descendant operator of conformal weight 2 , under the state-operator correspondence. ${ }^{10}$

\subsection{The Macdonald index}

The Macdonald index is the Macdonald limit, $p \rightarrow 0$, of the superconformal index. As the same set of operators, the Schur operators, contribute to the Macdonald index, it is also related to the chiral algebra, as a one-parameter $t$-refined version of the character. In [76], Song found that the quantum number $\ell=R+r$ in the Macdonald index

$$
\mathcal{I}(q, t)=\operatorname{tr}\left((-1)^{F} T^{\ell} q^{h}\right)
$$

where

$$
T:=t / q
$$

counts the number of fundamental generators in the chiral algebra used to obtain each state starting from the highest weight. A more detailed review on Song's work will be provided in section 2.9 .

\subsection{Argyres-Douglas superconformal field theories}

In the case of a weakly-coupled superconformal gauge theory with a Lagrangian description, one can write a matrix integral based on the field content of the gauge theory, and using that, evaluate the superconformal index [4]. An Argyres-Douglas theory is strongly-coupled and has no Lagrangian description. However, one can compute the superconformal index using the class $S$ theory construction, that is the compactification of $6 \mathrm{~d} \mathcal{N}=(2,0)$ theory on a Riemann surface with an irregular puncture, and compute the index using the TQFT defined on the Riemann surface [51, 75]. Further, in the case of rank-one Argyres-Douglas theories, it is not difficult to compute the index from BPS quivers [28] and the RG flow from $4 \mathrm{~d} \mathcal{N}=2 \mathrm{SYM}[1,61,62]$. In this work, we focus on Argyres-Douglas theories of type $\left(A_{n-1}, A_{m-1}\right), \operatorname{gcd}(n, m)=1$.

\subsection{TQFT approach to Macdonald index}

The Macdonald index of the class of theories we study in this article can be computed via the so-called TQFT approach as

$$
\mathcal{I}_{\left(A_{n-1}, A_{m-1}\right)}(q, t)=\sum_{\lambda} C_{\lambda}^{-1}(q, t) f_{\lambda}^{I_{n, m}}(q, t),
$$

where $\lambda=\left\{\lambda_{i}\right\}_{i=1}^{n-1}$ is a partition with $n-1$ rows, $C_{\lambda}$ is the 3 -pt coefficient in the TQFT picture

$$
C_{\lambda}^{-1}(q, t)=\frac{\tilde{P}_{\lambda}\left(t^{\rho} ; q, t\right)}{\prod_{i=1}^{r}\left(t^{d_{i}} ; q\right)_{\infty}}
$$

\footnotetext{
${ }^{10}$ A primary Schur operator is not necessarily primary under the action of the full $4 \mathrm{~d} \mathcal{N}=2$ superconformal algebra. For example, the R-symmetry current is a descendant of a scalar field.
} 
with $(a ; q)_{\infty}=\prod_{i=0}^{\infty}\left(1-a q^{i}\right), d_{i}$ is the degree of $i$-th Casimir in the Lie algebra $A_{n-1}$, $\tilde{P}_{\lambda}(x ; q, t)$ is the normalized Macdonald polynomial of $A_{n-1}$-type that satisfies

$$
\frac{1}{n !} \frac{(q ; q)^{n-1}}{(t ; q)^{n-1}} \oint \prod_{i} \frac{\mathrm{d} z_{i}}{2 \pi i z_{i}} \prod_{\alpha \in \Delta} \frac{\left(z^{\alpha} ; q\right)}{\left(t z^{\alpha} ; q\right)} \tilde{P}_{\lambda}(z ; q, t) \tilde{P}_{\mu}\left(z^{-1} ; q, t\right)=\delta_{\lambda \mu}
$$

and $f_{\lambda}^{I_{n, m}}$ is the wavefunction of the irregular puncture $I_{n, m}[75,85]$. For example, the wavefunction of $I_{2,2 i+1} i=1,2,3, \cdots$, is

$$
f_{\lambda}^{I_{2,2 i+1}}(q, t)=(-1)^{\frac{\lambda}{2}} q^{\frac{\lambda}{2}\left(\frac{\lambda}{2}+1\right)\left(i+\frac{3}{2}\right)}(t / q)^{\frac{\lambda}{2}(i+2)}\left(\frac{(t ; q)_{\frac{\lambda}{2}}\left(q^{\frac{\lambda}{2}+1} ; q\right)_{\frac{\lambda}{2}}\left(t^{2} q^{\lambda} ; q\right)}{(q ; q)_{\frac{\lambda}{2}}\left(t q^{\frac{\lambda}{2}} ; q\right)_{\frac{\lambda}{2}}\left(t q^{\lambda+1} ; q\right)}\right)^{\frac{1}{2}}
$$

where $\lambda$, a one-row partition, is even, and the wavefunction is zero when $\lambda$ is odd. For $I_{3, m}$, similarly, the wavefunction does not vanish only when the corresponding weight $\vec{w}$ of the representation $\lambda$ of $A_{2}$, that is $w_{1}=\lambda_{1}-\lambda_{2}, w_{2}=\lambda_{2}$, takes the form

$$
\left(w_{1}, w_{2}\right)=(3 k, 3 \ell), \quad \text { or } \quad\left(w_{1}, w_{2}\right)=(3 k-2,3 \ell-2),
$$

for some appropriate integers $k$ and $\ell$. For more details, refer to $[75,85]$.

Remark. When we expand the index with respect to $q$, the contribution from each $f_{\lambda}^{I_{n, m}}$ to the index starts from the level $(n+m) h(\lambda, n)$, where $h(\lambda, n)$ is a function that is independent of $m$. For example, $h(\lambda, 2)=\frac{\lambda}{4}\left(\frac{\lambda}{2}+1\right)$. In other words, if we truncate the index at for example $q^{10}$, the index for smaller $m$ contains more non-trivial information from the viewpoint of TQFT.

Remark. Following [85], the Macdonald index was shown to match exactly with the $t$ refined character of the vacuum and next-to-vacuum module in the large $m$ limit of $\left(p, p^{\prime}\right)=$ $(n, n+m)$ minimal models. This also motivates us to focus on the case of small $m$ in this work.

\subsection{Song's work}

In [76], Song showed that the Macdonald index of $\left(A_{1}, A_{2 k-2}\right)$ theory, which is dual to the $\mathcal{W}_{2}$ non-unitary $\left(p, p^{\prime}\right)=(2,2 k+3)$ minimal model $\mathcal{M}^{2,2 k+3}[28]$, is a $t$-refined character of Virasoro algebra that can be computed as follows. We first introduce the parameter $T:=t / q$. To each state in the module that can be written as

$$
L_{-i_{1}} L_{-i_{2}} \cdots L_{-i_{m}}|0\rangle, \quad \text { with } i_{1}+i_{2}+\cdots+i_{m}=h,
$$

we assign a weight $T^{m} q^{h}$, that is $\ell=m$ in (2.8), and the $t$-refined character is given by the sum of the contributions of all the states in the vacuum module of the dual chiral algebra. When there are null states in the module, we delete the states with largest $T$-weight from the spectrum.

To access non-vacuum modules from the gauge theory side, one needs to either insert defect operators in the perpendicular direction to the chiral algebra plane in 4d [29-31], or 
consider the lens space index of the gauge theory [40]. In the case of $\left(A_{n-1}, A_{m-1}\right)$ theories with $\operatorname{gcd}(n, m)=1$, the former approach is more powerful, and the correspondence between surface operators and non-vacuum modules of chiral algebra was worked out in [66].

The Macdonald indices in higher-rank cases and with surface operator inserted in $\left(A_{n-1}, A_{m-1}\right)$ theories with $\operatorname{gcd}(n, m)=1$ are computed in [85], via the TQFT approach and the Higgsing method, introduced in [53], to generate surface operators in gauge theory. We will not describe the details of the Higgsing method, but essentially what it does to the Macdonald index (2.10), in correspondence with inserting a surface operator (labeled by $a$ ) in the gauge theory, is to insert a factor of Macdonald polynomial. For example, for $n=2$

$$
\mathfrak{S}_{\lambda}^{a}(q, t)=q^{\frac{a}{2}} P_{(a)}\left(t^{\frac{1}{2}} q^{\frac{\lambda}{2}}\right)
$$

is inserted in the sum expression over $\lambda$. The study of Macdonald indices computed in this way suggests that the $t$-refined character in higher-rank $W_{N}$-algebras that reproduces the Macdonald index can be obtained as follows. Given a state generated from the highest weight state by $m_{j}$ spin- $j$ currents, $W^{(j)}$, we assign

$$
T^{\ell} q^{h}, \ell=\sum_{j=2}^{n} m_{j}
$$

to that state as its contribution, and we sum over all possible contributions to obtain the $t$-refined character.

In this work, we take a different approach, namely, we start from a statistical mechanics model described in the next section, define a natural $t$-refined character to it, and compare the result with the Macdonald index.

\section{Definitions. The statistical mechanics/combinatorics side}

We recall basic definitions from the statistical mechanics/combinatorics side

\subsection{Alternating-sign (bosonic) sum expressions of the Virasoro characters}

For a minimal Virasoro model labelled by $p, p^{\prime}, r, s, p<p^{\prime}, 0<r, p, 0<s<p^{\prime}$, the character can be written in the alternating-sign (Feigin-Fuchs) form

$$
\chi_{r, s}^{p, p^{\prime}}=\frac{1}{(q)_{\infty}} \sum_{\lambda=-\infty}^{\infty}\left(q^{\lambda^{2} p p^{\prime}+\lambda\left(p^{\prime} r-p s\right)}-q^{(\lambda p+r)\left(\lambda p^{\prime}+s\right)}\right),
$$

with $(q)_{\infty}=\prod_{i=1}^{\infty}\left(1-q^{i}\right)$, and the conformal dimension $\Delta_{r, s}^{p, p^{\prime}}$ is

$$
\Delta_{r, s}^{p, p^{\prime}}=\frac{\left(p^{\prime} r-p s\right)^{2}-\left(p^{\prime}-p\right)^{2}}{4 p p^{\prime}}
$$

Expression (3.1) for $\chi_{r, s}^{p, p^{\prime}}$ is related to the free-boson realization of the Virasoro algebra, and is known as a bosonic expression. For later purposes, it will be useful to note that $\chi_{r, s}^{p, p^{\prime}}=\chi_{p-r, p^{\prime}-s}^{p, p^{\prime}}$ and that $\left.\chi_{r, s}^{p, p^{\prime}}\right|_{q=0}=1$. 


\subsection{Constant-sign (fermionic) sum expressions of the Virasoro characters}

For $\mathcal{L}^{2,2 k+3}$, a constant-sign (fermionic) sum expression of the Virasoro characters is

$$
\chi_{r=1, s=a}^{2,2 k+3}(q)=\sum_{N_{1} \geq \cdots \geq N_{k} \geq 0} \frac{q^{N_{1}^{2}+\cdots+N_{k}^{2}+N_{a}+\cdots+N_{k}}}{(q)_{N_{1}-N_{2}} \cdots(q)_{N_{k-1}-N_{k}}(q)_{N_{k}}},
$$

where $|q|<1,(q)_{0}=1$ and $(q)_{n}=\prod_{i=1}^{n}\left(1-q^{i}\right)$ for $n>0$. Here $k \geq 1$ and $1 \leq a \leq k+1$. These are the expressions that we focus on in this work.

\subsection{Product expressions of the Virasoro characters}

The above fermionic character expressions (3.3) satisfy the Andrews-Gordon identities $[6,55]$

$$
\sum_{N_{1} \geq \cdots \geq N_{k} \geq 0} \frac{q^{N_{1}^{2}+\cdots+N_{k}^{2}+N_{a}+\cdots+N_{k}}}{(q)_{N_{1}-N_{2}} \cdots(q)_{N_{k-1}-N_{k}}(q)_{N_{k}}}=\prod_{\substack{n=1 \\ n \neq 0, \pm i(\bmod 2 k+3)}}^{\infty} \frac{1}{1-q^{n}}
$$

The $k=1$ cases are the Rogers-Ramanujan identities [71-73].

\subsection{The work of Bressoud}

In [22], Bressoud interpreted the fermionic sum expression (3.3) as the character of Dyck paths with fixed initial and end points. This interpretation works only in the case of $\mathcal{L}^{2,2 k+3}$ models, $k=1,2, \cdots$. An equivalent intrpretation, also in terms of Dyck paths, developed in [48], extends to all $\left(p, p^{\prime}\right)$ Virasoro minimal models. In this work, we use the paths of [48], a review of which is in the next subsection.

\subsection{The paths of Virasoro minimal model characters. The vacuum modules}

One can express a Virasoro minimal model character as the generating function of weighted Dyck paths that connect two given points on a restricted-height semi-infinite lattice. More precisely, for a $\left(p, p^{\prime}\right)$ model, one prepares a lattice which is $p^{\prime}-1$ bands in height, and $L+2$ bands in length, and considers Dyck paths that connect the points $\left\{\left(i, h_{i}\right)\right\}_{i=0}^{L+1}$, and satisfy

- $h_{0}=a, h_{L}=b, h_{L+1}=c(c=b \pm 1)$,

- $h_{i+1}=h_{i} \pm 1$

The correspondence with the Virasoro minimal model characters is obtained by choosing the labels $(r, s)$ of the characters such that $s=a$, and $r$ is

$$
r=\left\lfloor p c / p^{\prime}\right\rfloor+\frac{b-c+1}{2}
$$

A ground-state band is defined as a band between $j$-th line and $(j+1)$-th line, such that

$$
\left\lfloor j p / p^{\prime}\right\rfloor \neq\left\lfloor(j+1) p / p^{\prime}\right\rfloor
$$


For example, in the $\mathcal{L}^{2,5}$ Lee-Yang model, we have a $4 \times(L+2)$-lattice (see figure 1 ) and the ground-state band lies between the 2nd and the 3rd lines in the lattice. Further, we need to assign a coordinate system $(x, y)$ with

$$
x_{i}=\frac{i-\left(h_{i}-a\right)}{2}, \quad y_{i}=\frac{i+\left(h_{i}-a\right)}{2},
$$

to each point $\left(i, h_{i}\right)$.

\subsubsection{The weight of a path}

To each point at $\left(i, h_{i}\right)(i=1,2, \cdots, L)$, we assign a weight $c_{i}$ that depends on the shape of the path connecting the point with its neighbors and the position of the point. When the path enters a ground-state band in the upward direction at point $\left(i, h_{i}\right)$ (figure $2(\mathrm{a})$ ), or it reaches a peak at $\left(i, h_{i}\right)$ outside the ground-state band (figure $2(\mathrm{~b})$ ), the weight $c_{i}$ is determined by

$$
c_{i}=x_{i}
$$

When the path enters in the downward direction to the ground-state band (figure 3 (a)) or hits a valley outside the ground-state band (figure 3 (b)), we assign it

$$
c_{i}=y_{i}
$$

Otherwise, the weight is set to zero. The weight of the whole path is given by the sum of the weights of each point,

$$
w t(P)=\sum_{i=1}^{L} c_{i}
$$

The 'L-finite' (or 'finitized') character for a fixed-length lattice and fixed parameters $(a, b, c)$, labeling the start and end points, is given by the sum over all allowed finite-length weighted paths $P$,

$$
\chi_{a, b, c}^{p, p^{\prime}}(L, q)=\sum_{P} q^{w t(P)}
$$

The character of the corresponding minimal model is obtained in the limit $L \rightarrow \infty$,

$$
\chi_{r, s=a}^{p, p^{\prime}}(q)=\lim _{L \rightarrow \infty} \chi_{a, b, c}^{p, p^{\prime}}(L, q)
$$

The vacuum module in an $\mathcal{L}^{2,2 k+3}$ model is characterized by $r=s=a=1$, and $r$ is fixed by $b$ and $c$ through (3.5). In principle, there are two equivalent combinations ${ }^{11}$ of $(b, c)$ that give the same value of $r$. We choose the one such that the lattice square spanned by the point $(L, b)$ and $(L+1, c)$ is contained inside the ground-state band. This fixes $b=2$ and $c=3$ in the Lee-Yang model $\mathcal{L}^{2,5}$, as shown in figure 1 . More generally, in the case of the models $\mathcal{L}^{2,2 k+3}, b=k+1$ and $c=k+2$.

\footnotetext{
${ }^{11}$ Equivalent in the sense that the resulting character is the same.
} 


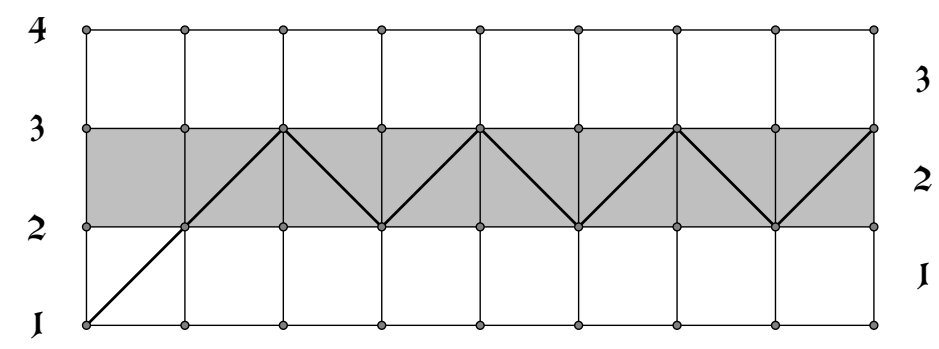

Figure 1. A minimal path in the vacuum module of the restricted solid-on-solid model $\mathcal{L}^{p, p^{\prime}}=\mathcal{L}^{2,5}$, labeled by $a=1, b=2$, and $c=3$. The numbers on the left label the possible initial points of paths, and correspond to the label $s$ in $\chi_{r, s}^{p, p^{\prime}}=\chi_{r, s}^{2,5}$. The numbers on the right label the possible locations of the ground-state (shaded) bands that paths can end up oscillating in. The number of ground-state bands is the range of the label $r$ in $\chi_{r, s}^{p, p^{\prime}}=\chi_{r, s}^{2,5}$. In this example, there is only one ground state band, and $\chi_{r, s}^{p, p^{\prime}}=\chi_{1,1}^{2,5}$.

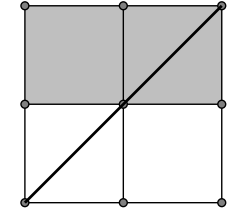

$(a)$

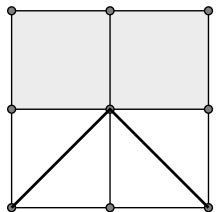

(b)

Figure 2. The two cases where we assign the weight $c_{i}=x_{i}$ to the middle point located at position $i$. Scanning from left to right, (a) the path enters the ground-state (shaded) band from a non-ground-state (white) band below at the middle point, (b) the path reaches a peak at the middle point outside the ground-state (shaded) band. A lightly-shaded band (the upper band in figure (b)) can be a ground-state band or not.

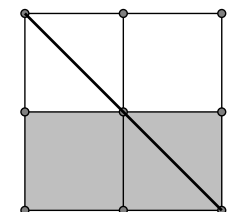

(a)

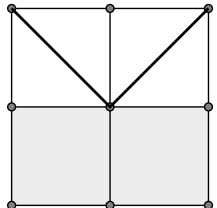

(b)

Figure 3. The two cases where we assign the weight $c_{i}=y_{i}$ to the middle point located at position $i$. Scanning from left to right, (a) the path enters the ground-state (shaded) band from the top at the middle point, (b) the path makes a valley at the middle point in a non-ground-state (white) band. The lightly-shaded band (the lower band in figure (b)) can be either a ground-state band or not.

Remark. In the case of the models $\mathcal{L}^{2,2 k+3}$ (and only in this case), one can check that the contributions to the weight of a path come effectively from the positions of the peaks and valleys (that is, the positions along the horizontal extension of the lattice, which starts from $i=0$ ), outside the ground-state band, and that it costs nothing to wander inside the ground-state band. A typical path with finite weight (in the $L \rightarrow \infty$ limit) will converge into a zigzag inside the ground-state band at finite $i$. 


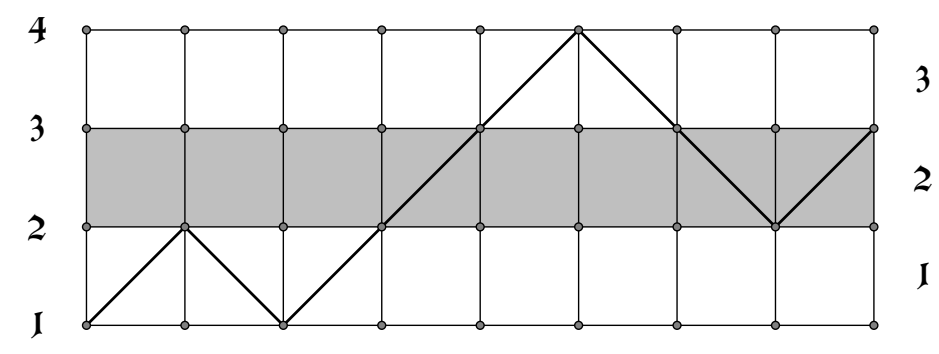

Figure 4. A non-minimal path in the restricted solid-on-solid model $\mathcal{L}^{p, p^{\prime}}=\mathcal{L}^{2,5}$, labeled by $a=1, b=2$, and $c=3$.

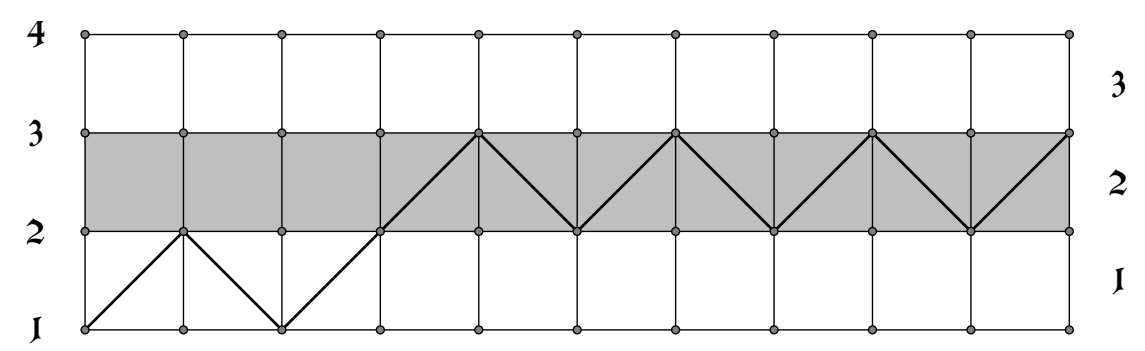

Figure 5. A non-minimal path in the restricted solid-on-solid model $\mathcal{L}^{2,5}$, labeled by $a=1, b=2$, and $c=3$, that contains a valley at $(x, y)=(1,1)$ of weight $w t=1+1=2$.

Remark. The path with no peaks or valleys outside the ground-state band is the minimal path (an example is in figure 1), and corresponds to the highest weight state in the corresponding minimal model module. (Virasoro) Descendant states correspond to nonminimal paths.

An example of a non-minimal path in the Lee-Yang model $\mathcal{L}^{2,5}$ is in figure 4. Each peak and valley outside the ground-state band is assigned a definite weight. For example, the path with a single valley of weight 2 and the path with a single valley of weight 4 in the Lee-Yang model $\mathcal{L}^{2,5}$ are shown in figure 5 and 6 , respectively. We can also consider a path with both valleys, as in figure 7 , whose weight is $w t=2+4=6$. The character of the vacuum module for the Lee-Yang model $(k=1)$ can then be computed as

$$
\sum_{N_{1} \geqslant 0} \sum_{\substack{t_{1}, t_{2}, \cdots, t_{N_{1}} \\ t_{i+1}-t_{i} \geqslant 2, t_{1} \geqslant 2}} q^{t_{1}+t_{2}+\cdots+t_{N_{1}}}=\sum_{N_{1} \geqslant 0} \frac{q^{N_{1}^{2}+N_{1}}}{(q)_{N_{1}}},
$$

where $N_{1}$ gives the number of valleys plus peaks, and $t_{i}$ denotes the corresponding weight of the $i$-th valley or peak. ${ }^{12}$ These peaks and valleys behave as excitations of (quasi-)particles, and we refer to them as particles.

\subsubsection{Higher- $k$ models}

Models with higher $k$ are built using the same rules described in the previous subsection, but they are naturally somewhat more complicated. Consider $k=2$, that is $p=2, p^{\prime}=7$. The lattice in figure 8 , of size $6 \times(L+2), L=12$, shows the minimal path in this model.

\footnotetext{
${ }^{12}$ This computation is explained in detail in [48].
} 


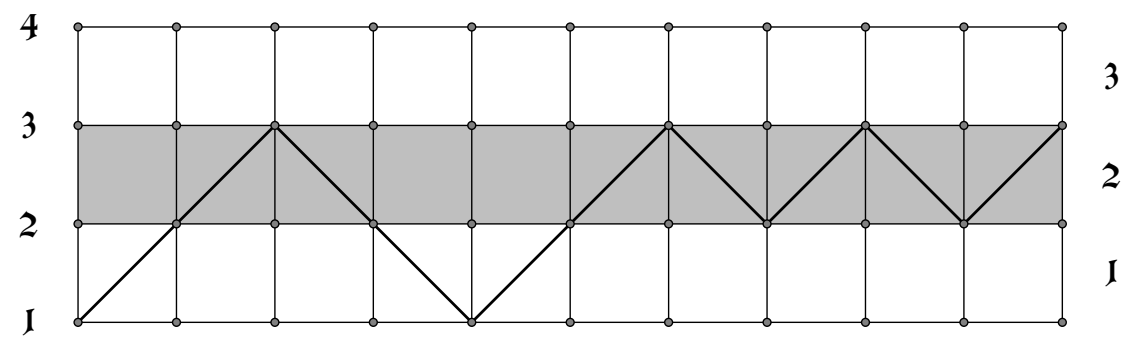

Figure 6. A non-minimal path in the Lee-Yang restricted solid-on-solid model $\mathcal{L}^{2,5}$, labeled by $a=1, b=2$, and $c=3$, that contains a valley at $(x, y)=(2,2)$ of weight $w t=2+2=4$.

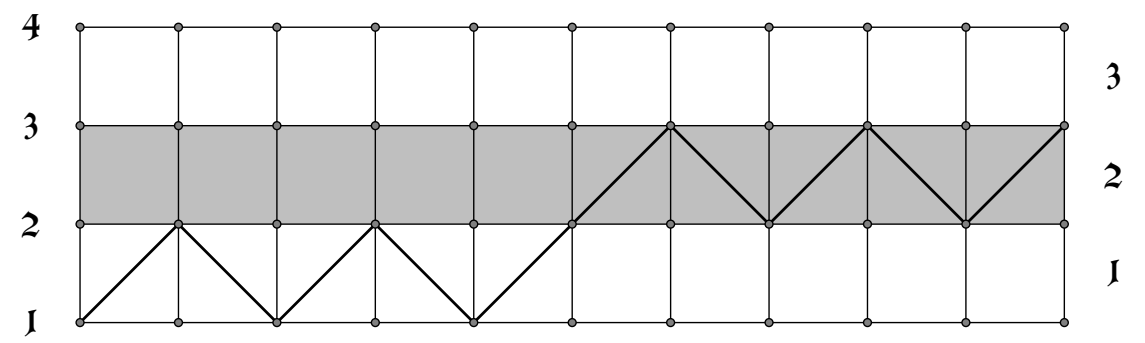

Figure 7. A non-minimal path in the Lee-Yang restricted solid-on-solid model $\mathcal{L}^{2,5}$, labeled by $a=1, b=2$, and $c=3$, that contains a valley at $(x, y)=(1,1)$ and another valley at $(2,2)$ of weight $w t=1+1+2+2=6$.

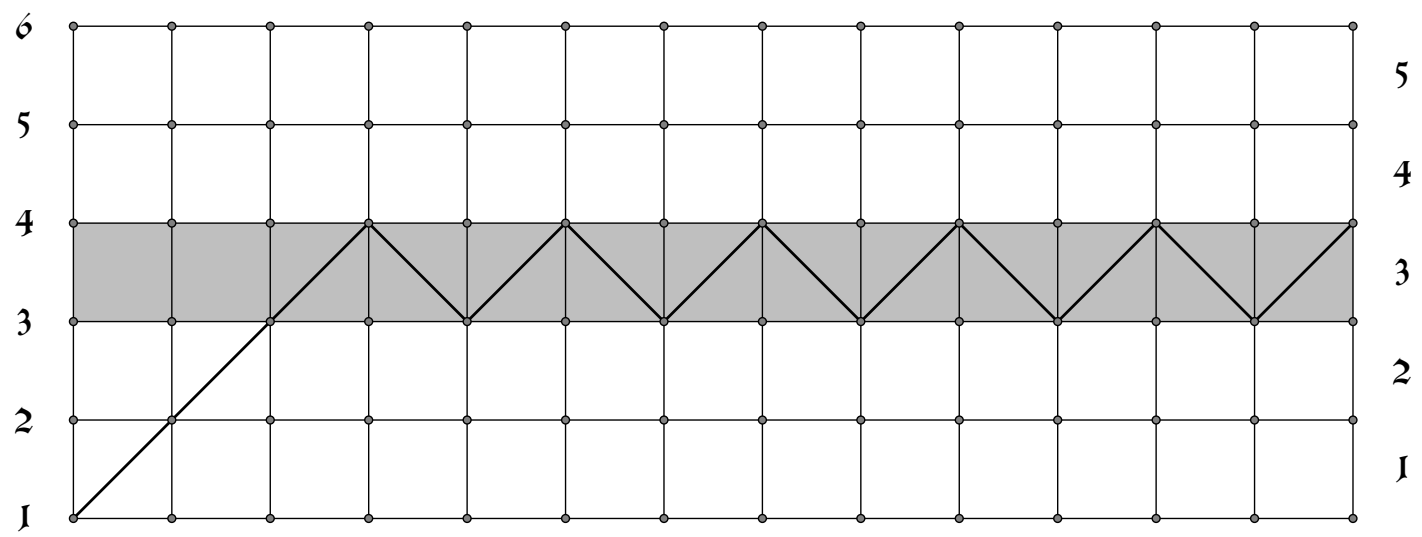

Figure 8. The minimal path in the vacuum module of the restricted solid-on-solid model $\mathcal{L}^{2,7}$, labeled by $a=1, b=3$, and $c=4$. The weight of a minimal path is zero.

For higher $k$, there are $k$ particle species. For $k=2$, there are two different paths, one in figure 9 and one in 10, with a single particle each, of different particle species, but the same weight, 4 .

Remark. We note that one can judge the type of a given particle by using the moves defined in [44] to transform it to the particle with minimal weight of the same type.

Remark. For $k=2,3, \cdots$, there is a $\mathbb{Z}_{2}$ reflection symmetry between the peaks and valleys with the same weight. This symmetry is clear when we compare figures 9 and 10 with weight-4, and the reflection symmetry is with respect to the lower boundary of the groundstate band. Similarly, when we compare figure 11 and 12, with weight-5, and the reflection 


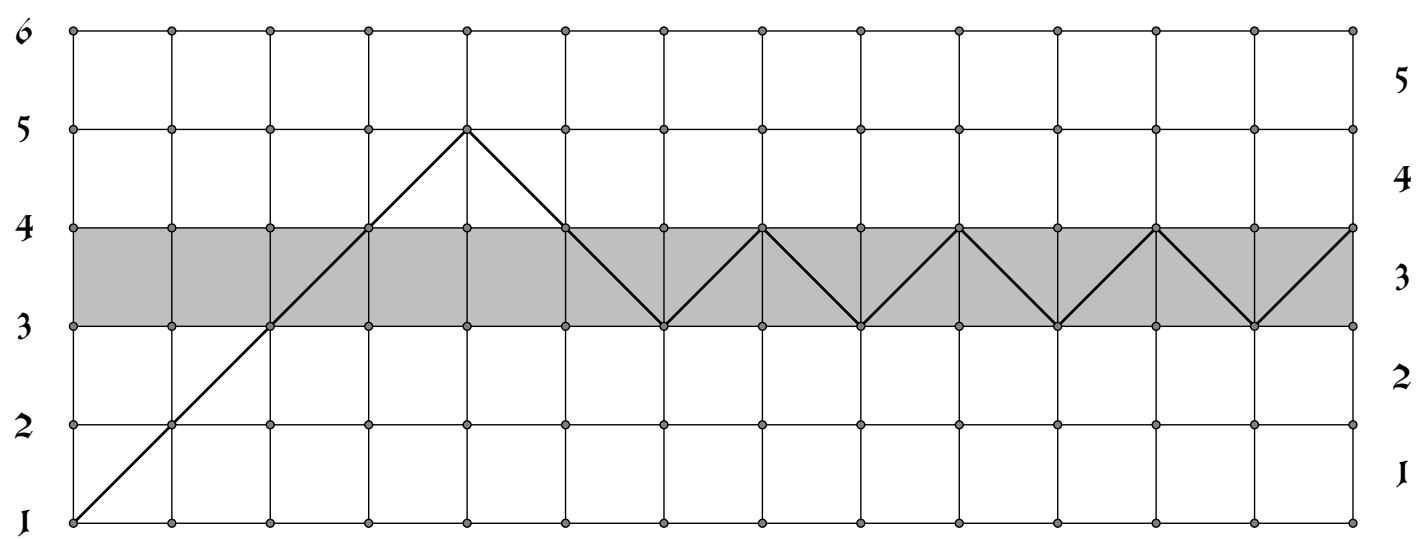

Figure 9. A non-minimal path in the restricted solid-on-solid model $\mathcal{L}^{2,7}$ with a peak at $(x, y)=$ $(0,4)$ of weight 4 .

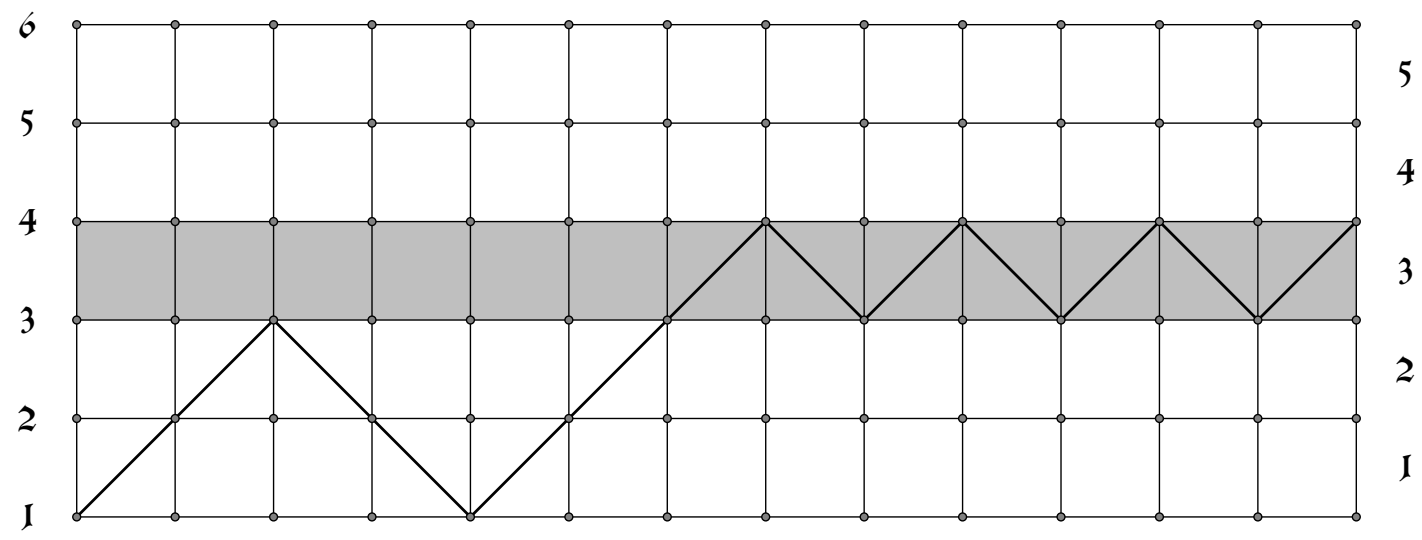

Figure 10. A non-minimal path in the restricted solid-on-solid model $\mathcal{L}^{2,7}$, with a valley at $(x, y)=(2,2)$ of weight 4 . The valley between the 3 -rd vertical line (at $i=2)$ and the 7 -th vertical line (at $i=6$ ) is obtained by reflecting with respect to the lower boundary of the ground-state band from the peak in figure 9 .

symmetry is with respect to the upper boundary of the ground-state band. For $k>2$, we have more than two types of particles, and there will be a $\mathbb{Z}_{2}$ reflection symmetry between each pair of two different species. We will interpret these quasi-particles as BPS operators in the context of gauge theory, however, it is not clear what kind of role these $\mathbb{Z}_{2}$ reflection symmetries play there.

The constant-sign sum expression of the $k=2$ vacuum character is

$$
\sum_{N_{1} \geqslant N_{2} \geqslant 0} \frac{q^{N_{1}^{2}+N_{2}^{2}+N_{1}+N_{2}}}{(q)_{N_{1}-N_{2}}(q)_{N_{2}}}=1+\frac{q^{2}}{1-q}+\frac{q^{4}}{1-q}+\frac{q^{6}}{(1-q)\left(1-q^{2}\right)}+\frac{q^{8}}{(1-q)^{2}}+\cdots
$$

The term $\frac{q^{2}}{1-q}$ represents the contributions from all paths with a single valley (such as the paths in figure 10 and 12). The term $\frac{q^{4}}{1-q}$, however, comes from the contributions of all paths with a single peak of weight larger than 3 (such as the paths in figure 9 and 11). 


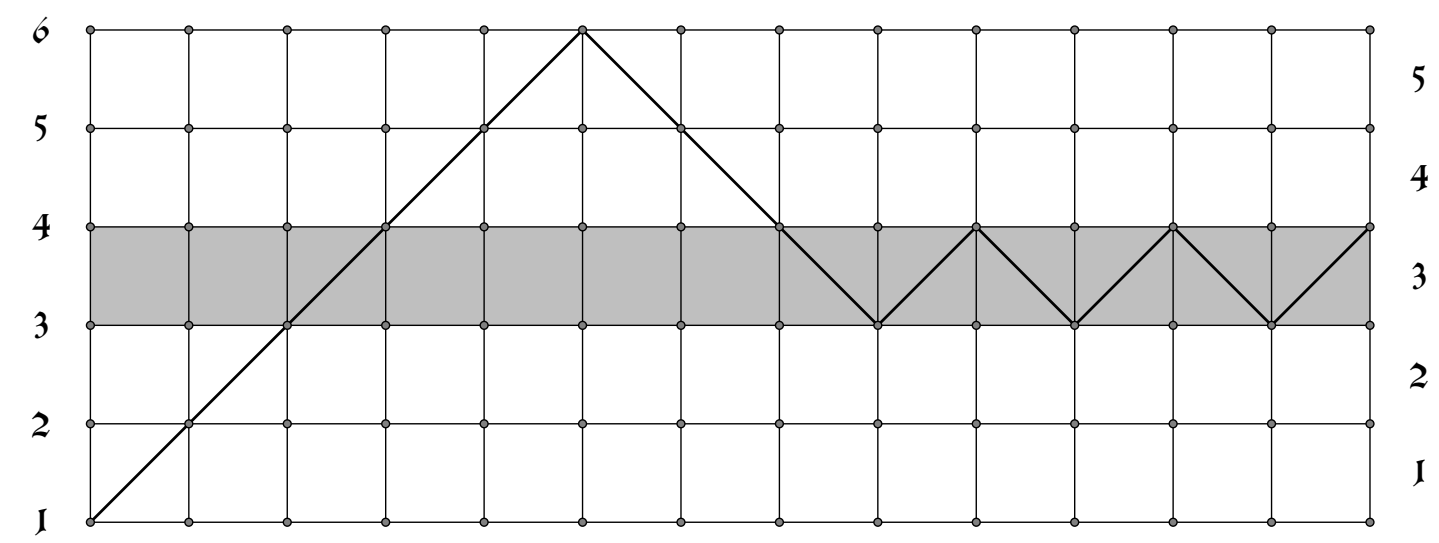

Figure 11. A non-minimal path in the restricted solid-on-solid model $\mathcal{L}^{2,7}$ with a peak at $(x, y)=$ $(0,5)$ of weight 5 .

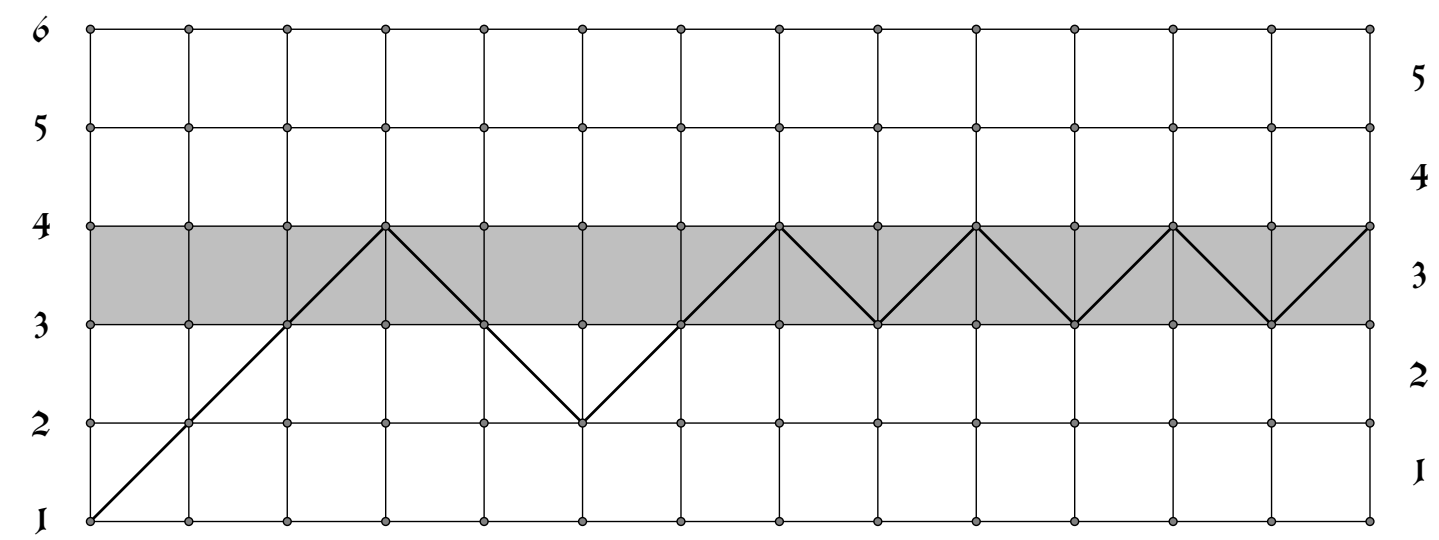

Figure 12. A non-minimal path in the restricted solid-on-solid model $\mathcal{L}_{2,7}$. The valley between the 4 -th vertical line (position $i=3$ ) and the 8 -th vertical line (position $i=7$ ) is obtained by reflecting the peak in figure 11 with respect to the upper boundary of the ground-state band.

The term $\frac{q^{6}}{(1-q)\left(1-q^{2}\right)}$ and $\frac{q^{8}}{(1-q)^{2}}$ can thus be interpreted respectively as the contributions from paths with two valleys and paths with one peak and one valley. In this way, we see that $N_{1}$ in this example counts the number of all particles, while $N_{2}$ counts the number of particles of the same type as those in figure 9 and 11, that's is particles of height 1 above the ground-state band.

In the $\mathcal{L}^{2,2 k+3}$ model, we can have $k$ types of peaks/valleys with the same weight. $N_{1}$ always counts the total number of all particles, and $N_{i}, i>1$ counts the number of different particle species. For example, the three paths with weight 6 , in the case $k=3$, are shown in different colors in figure 13.

\subsection{The paths of constant-sign Virasoro characters. The next-to-vacuum mod- ules}

To go to non-vacuum modules, we change $a$ to values larger than 1 . The next-to-vacuum module corresponds to $a=2$. For example in the Lee-Yang model $\mathcal{L}^{2,5}$, the corresponding 


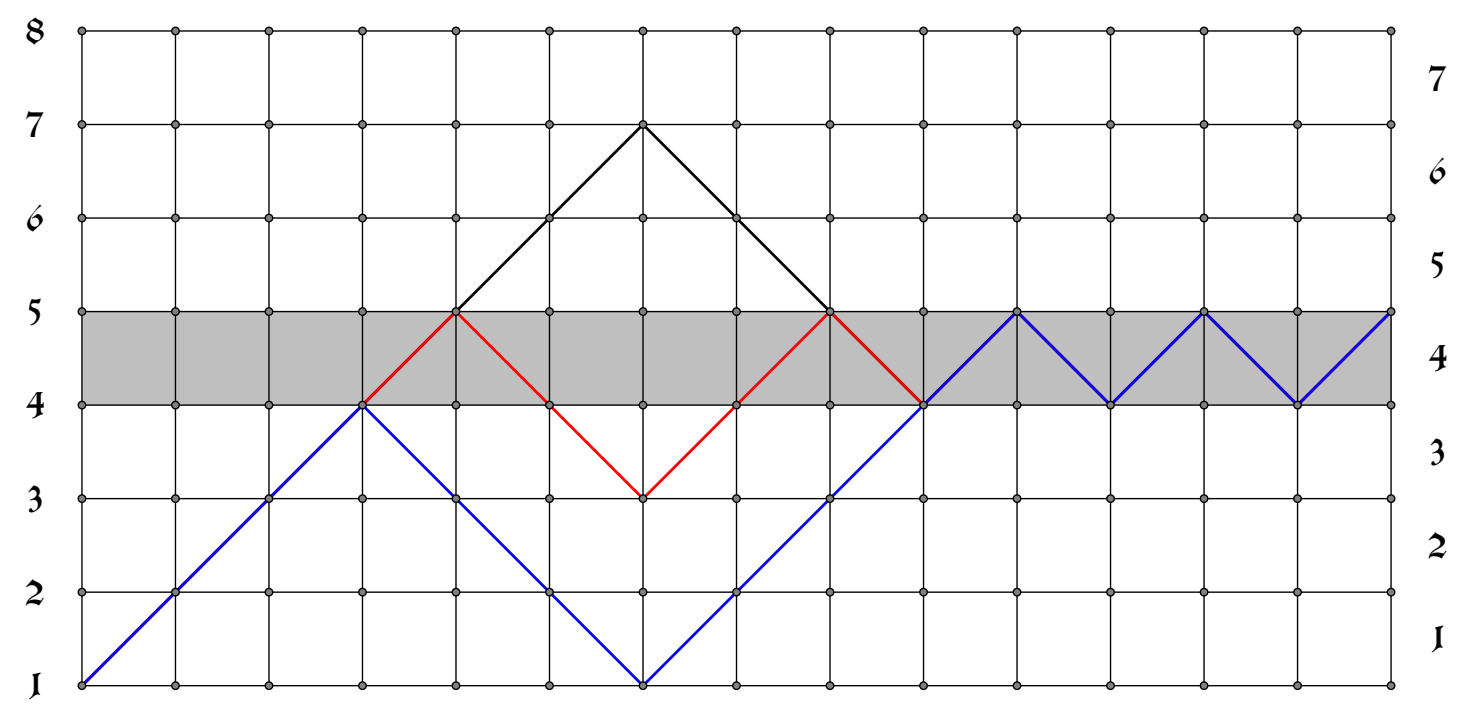

Figure 13. Three paths with single peak/valley in the restricted solid-on-solid model $\mathcal{L}^{2,9}$ respectively shown in black, red and blue colors.

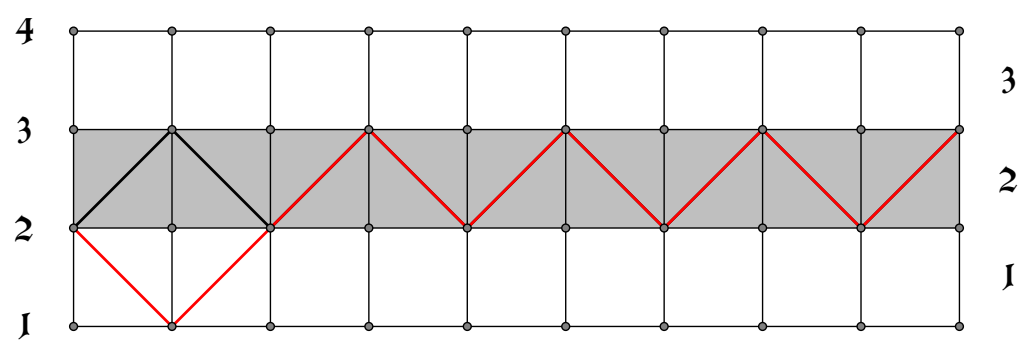

Figure 14. The minimal path (black) and a path with weight 1 (red) in the next-to-vacuum module of the Lee-Yang restricted solid-on-solid model $\mathcal{L}^{2,5}$, labeled by $a=2, b=2$, and $c=3$.

primary Virasoro field has conformal dimension $\Delta=-\frac{1}{5}$. The minimal path (with weight zero) that corresponds to the highest weight state is shown as the black line in figure 14 . A direct consequence of the changing value of $a$ is the appearance of new particle configurations with weight 1 (see the red path shown in figure 14). The character of this module is then modified to

$$
\sum_{N_{1} \geqslant 0} \sum_{\begin{array}{c}
t_{1}, t_{2}, \cdots, t_{N_{1}} \\
t_{i+1}-t_{i} \geqslant 2, t_{1} \geqslant 1
\end{array}} q^{t_{1}+t_{2}+\cdots+t_{N_{1}}}=\sum_{N_{1} \geqslant 0} \frac{q^{N_{1}^{2}}}{(q)_{N_{1}}}
$$

where $N_{1}$ again counts the number of all peaks and valleys.

\subsubsection{Higher- $k$ models}

A similar analysis extends to higher- $k$ models. We take $k=2$ as an example again. Here, we also have a possible new valley of weight 1 (see the red path in figure 15), and there are two types of particles (two paths with the same weight $w t=3$ are shown in figure 15 


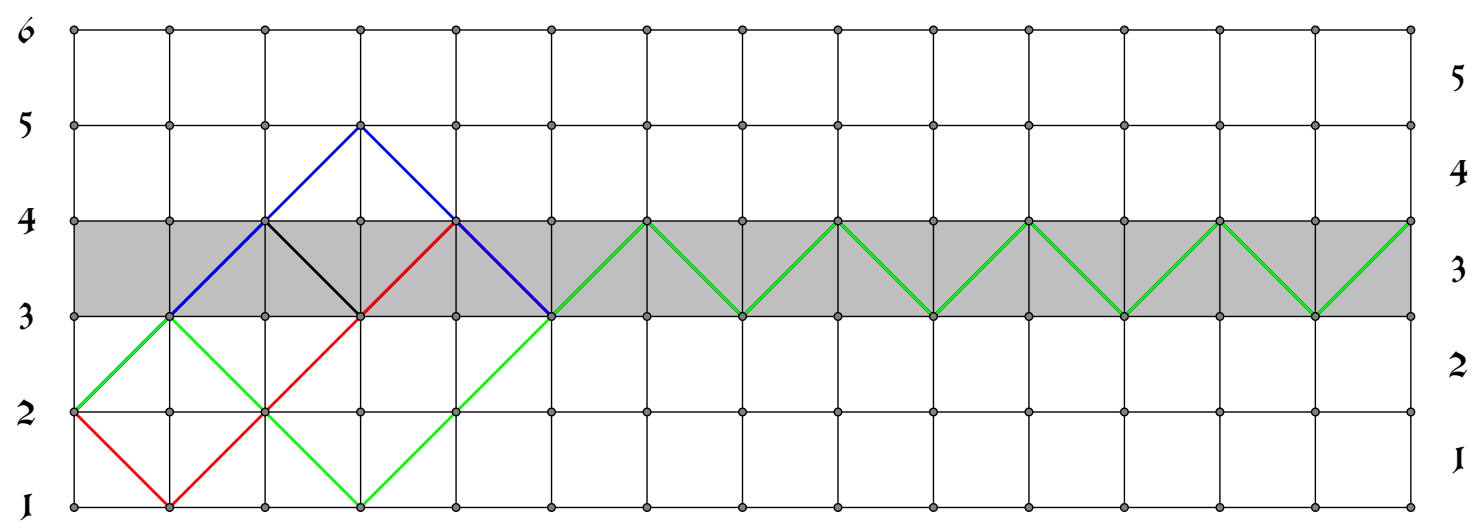

Figure 15. Several paths in the next-to-vacuum module of the restricted solid-on-solid model $\mathcal{L}^{2,7}$, labeled by $a=2, b=3$, and $c=4$. The minimal path in black, the path with weight 1 in red, and two paths with weight 3 in blue and green.

in blue and green). These modifications are reflected in the constant-sign sum expression for the character

$$
\sum_{N_{1} \geqslant N_{2} \geqslant 0} \frac{q^{N_{1}^{2}+N_{2}^{2}+N_{2}}}{(q)_{N_{1}-N_{2}}(q)_{N_{2}}}=1+\frac{q}{1-q}+\frac{q^{3}}{1-q}+\cdots
$$

It still holds that $N_{1}$ counts the total number of particles, and $N_{2}$ counts the number of particles of the type shown in blue in figure 15 .

\subsection{A $T$-refinement of the constant-sign sum expressions of the Virasoro char- acters as Macdonald indices}

Since there are $k$ particle species (as peaks or valleys) in the $\mathcal{L}^{2,2 k+3}$ model, a natural $t$-refined counting assigns a power of the refinement parameter $T$ to each particle, where $T=t / q$. In the Lee-Yang model $(k=1)$, there is only one type of particles, so we assign a weight $T$ to each particle in a path, and then the $t$-refined characters (written in terms of $T$ ) of the vacuum module and the next-to-vacuum module are

$$
\chi_{a=1}^{(2,5)}(q, T)=\sum_{N_{1} \geqslant 0} \frac{q^{N_{1}^{2}+N_{1}}}{(q)_{N_{1}}} T^{N_{1}}, \quad \chi_{a=2}^{(2,5)}(q, T)=\sum_{N_{1} \geqslant 0} \frac{q^{N_{1}^{2}}}{(q)_{N_{1}}} T^{N_{1}}
$$

In the case of $k=3$, there are two types of (excited) particles with weight larger than 3 in the vacuum module, and two in the next-to-vacuum module. We assign the weight $T$ to the first type that is counted by $N_{1}-N_{2}$ (such as the valley in figure 12 , and the valley in green in figure 15), and $T^{2}$ to the other type counted by $N_{2}$ (such as the peak in figure 11 and the peak in blue in figure 15). The $t$-refined character formulas are thus given by

$$
\begin{aligned}
& \chi_{a=1}^{(2,7)}(q, T)=\sum_{N_{1} \geqslant N_{2} \geqslant 0} \frac{q^{N_{1}^{2}+N_{2}^{2}+N_{1}+N_{2}}}{(q)_{N_{1}-N_{2}}(q)_{N_{2}}} T^{N_{1}+N_{2}}, \\
& \chi_{a=2}^{(2,7)}(q, T)=\sum_{N_{1} \geqslant N_{2} \geqslant 0} \frac{q^{N_{1}^{2}+N_{2}^{2}+N_{2}}}{(q)_{N_{1}-N_{2}}(q)_{N_{2}}} T^{N_{1}+N_{2}} .
\end{aligned}
$$


We see in this way that in general we can refine the character with the factor

$$
T^{\sum_{i=1}^{k} N_{i}}
$$

in the sum expression, that is to say, each particle of the $i$-th type, whose number is counted by $N_{i}-N_{i+1}$ (with $N_{k+1} \equiv 0$ ), is assigned a weight $T^{i}$. We remark that $\sum_{i=1}^{k} N_{i}$ is the linear part of the power of $q$, that is, $\sum_{i} N_{i}^{2}+N_{i}$, in the constant sign sum expression for the vacuum character. We will see from the series expansion of the sum expression that the above prescription matches Song's prescription to refine the Schur index to the Macdonald index, which also matches the computation of the Macdonald index from the TQFT approach.

\section{A proposal for a closed-form expression for the Macdonald index}

We give our main proposals in the form of three conjectures and provide evidence for them.

\subsection{Main proposal}

Recall that the $q$-series identities of Andrews-Gordon $[6,55]$ take the form

$$
\chi_{a}^{(2,2 k+3)}(q)=\sum_{N_{1} \geq \cdots \geq N_{k} \geq 0} \frac{q^{N_{1}^{2}+\cdots+N_{k}^{2}+N_{a}+\cdots+N_{k}}}{(q)_{N_{1}-N_{2}} \cdots(q)_{N_{k-1}-N_{k}}(q)_{N_{k}}}=\prod_{\substack{n=1 \\ n \neq 0, \pm i(\bmod 2 k+3)}}^{\infty} \frac{1}{1-q^{n}}
$$

where $|q|<1, k \geq 1$ and $1 \leq a \leq k+1$. We have already seen that $N_{i}$ 's for $i=$ $1, \cdots, k$ count the number of particles of different species in the paths approach. The $t$ refined version of the character (4.1), following the prescription we described in the previous section, then is

$$
\chi_{a}^{(2,2 k+3)}(q, T)=\sum_{N_{1} \geq \cdots \geq N_{k} \geq 0} \frac{q^{N_{1}^{2}+\cdots+N_{k}^{2}+N_{a}+\cdots+N_{k}}}{(q)_{N_{1}-N_{2}} \cdots(q)_{N_{k-1}-N_{k}}(q)_{N_{k}}} T^{\sum_{i=1}^{k} N_{i}}
$$

We first conjecture that the $t$-refined characters of the vacuum and next-to-vacuum modules are equal to the corresponding Macdonald indices for $n=2$.

\subsubsection{Conjecture 1}

$$
\begin{aligned}
& \chi_{a=0}^{(2,2 k+3)}(q, T)=\mathcal{I}_{\left(A_{1}, A_{2 k}\right)}(q, t)=\sum_{\lambda} C_{\lambda}^{-1}(q, t) f_{\lambda}^{I_{2,2 k+1}}(q, t), \\
& \chi_{a=1}^{(2,2 k+3)}(q, T)=\mathcal{I}_{\left(A_{1}, A_{2 k}\right)}^{\mathbb{S}^{1}}(q, t)=\sum_{\lambda} C_{\lambda}^{-1}(q, t) f_{\lambda}^{I_{2,2 k+1}}(q, t) \mathfrak{S}_{\lambda}^{1}(q, t)
\end{aligned}
$$

As there are series of fermionic sum expressions for characters of $\mathcal{W}_{3}$ model with $\left(p, p^{\prime}\right)=(3,7)$, we conjecture that the $t$-refined version of these expressions for the vacuum and next-to-vacuum modules agree with the corresponding Macdonald indices. 


\subsubsection{Conjecture 2}

$$
\begin{aligned}
\chi_{\left(s_{1}, s_{2}\right)}^{(3,7)} & =(1,1) \\
& =\sum_{n_{1}, n_{2}, n_{3}, n_{4} \geqslant 0} \frac{q^{\left(n_{1}+n_{2}+n_{3}\right)^{2}+\left(n_{2}+n_{3}\right)^{2}+n_{3}{ }^{2}+n_{4}{ }^{2}+\left(n_{1}+2 n_{2}+3 n_{3}\right) n_{4}+\left(n_{1}+2 n_{2}+3 n_{3}+2 n_{4}\right)}}{(q)_{n_{1}}(q)_{n_{2}}(q)_{n_{3}}(q)_{n_{4}}} T^{n_{1}+2 n_{2}+3 n_{3}+2 n_{4}} \\
& =\mathcal{I}_{\left(A_{2}, A_{3}\right)}(q, t) \\
& =\sum_{\lambda} C_{\lambda}^{-1}(q, t) f_{\lambda}^{I_{3,4}}(q, t), \\
\chi_{\left(s_{1}, s_{2}\right)}^{(3,7)} & =(1,2) \\
& =\sum_{n_{1}, n_{2}, n_{3}, n_{4} \geqslant 0} \frac{q^{\left(n_{1}+n_{2}+n_{3}\right)^{2}+\left(n_{2}+n_{3}\right)^{2}+n_{3}^{2}+n_{4}^{2}+\left(n_{1}+2 n_{2}+3 n_{3}\right) n_{4}+\left(n_{2}+2 n_{3}+n_{4}\right)}}{(q)_{n_{1}}(q)_{n_{2}}(q)_{n_{3}}(q) n_{n_{4}}} T^{n_{1}+2 n_{2}+3 n_{3}+2 n_{4}} \\
& =\mathcal{I}_{\left(A_{2}, A_{3}\right)}^{\mathbb{S} 1,0}(q, t) \\
& =\sum_{\lambda} C_{\lambda}^{-1}(q, t) f_{\lambda}^{I_{3,4}}(q, t) \mathbb{S}_{\lambda}^{1,0}(q, t)
\end{aligned}
$$

We provide evidence for the above conjectures by comparing both sides as series expansions in $q$ (where $t=T q$ ) and matching them up to high orders.

We further push this correspondence to interpret these particles as BPS operators contributing to the Schur/Macdonald index.

\subsubsection{Conjecture 3: a path interpretation of aspects of the Schur index}

- The number of types of primary Schur operators is the number of particles. ${ }^{13}$

- Each path corresponds to a composite operator. Each particle in a path corresponds to a Schur operator.

- A particle at minimal position (smallest possible weight) corresponds to a primary Schur operator. A particle far from a minimal position corresponds to a derivative of a primary Schur operator, that is, a descendant Schur operator, ${ }^{14}$ the distance from the minimal position equals the number of derivatives.

\subsection{The Macdonald version of the sum expressions of the Virasoro characters}

The expression for the $t$-refined Virasoro character is

$$
\chi_{a}^{(2,2 k+3)}(q, T)=\sum_{N_{1} \geq \cdots \geq N_{k} \geq 0} \frac{q^{N_{1}^{2}+\cdots+N_{k}^{2}+N_{a}+\cdots+N_{k}}}{(q)_{N_{1}-N_{2}} \cdots(q)_{N_{k-1}-N_{k}}(q)_{N_{k}}} T^{N_{1}+\cdots+N_{k}} .
$$

\footnotetext{
${ }^{13}$ For definition of primary Schur operators, see 2.5.

${ }^{14}$ For definition of descendant Schur operators, see 2.5
} 
Let us list the $t$-refined characters of the vacuum module and the next-to-vacuum module for $k=1,2,3$ as a series expansion in $q$.

$$
\begin{aligned}
& \chi_{a=1}^{(2,5)}(q, T)=\sum_{N_{1} \geqslant 0} \frac{q^{N_{1}^{2}+N_{1}}}{(q)_{N_{1}}} T^{N_{1}} \\
& =1+T q^{2}+T q^{3}+T q^{4}+T q^{5}+\left(T+T^{2}\right) q^{6}+\left(T+T^{2}\right) q^{7} \\
& +\left(T+2 T^{2}\right) q^{8}+\left(T+2 T^{2}\right) q^{9}+\left(T+3 T^{2}\right) q^{10}+\mathcal{O}\left(q^{11}\right), \\
& \chi_{a=2}^{(2,5)}(q, T)=\sum_{N_{1} \geqslant 0} \frac{q^{N_{1}^{2}}}{(q)_{N_{1}}} T^{N_{1}} \\
& =1+T q+T q^{2}+T q^{3}+\left(T+T^{2}\right) q^{4}+\left(T+T^{2}\right) q^{5}+\left(T+2 T^{2}\right) q^{6} \\
& +\left(T+2 T^{2}\right) q^{7}+\left(T+3 T^{2}\right) q^{8}+\left(T+3 T^{2}+T^{3}\right) q^{9} \\
& +\left(T+4 T^{2}+T^{3}\right) q^{10}+\mathcal{O}\left(q^{11}\right), \\
& \chi_{a=1}^{(2,7)}(q, T)=\sum_{N_{1} \geqslant N_{2} \geqslant 0} \frac{q^{N_{1}^{2}+N_{2}^{2}+N_{1}+N_{2}}}{(q)_{N_{1}-N_{2}}(q)_{N_{2}}} T^{N_{1}+N_{2}} \\
& =1+T q^{2}+T q^{3}+\left(T+T^{2}\right) q^{4}+\left(T+T^{2}\right) q^{5}+\left(T+2 T^{2}\right) q^{6} \\
& +\left(T+2 T^{2}\right) q^{7}+\left(T+3 T^{2}+T^{3}\right) q^{8}+\left(T+3 T^{2}+2 T^{3}\right) q^{9} \\
& +\left(T+4 T^{2}+3 T^{3}\right) q^{10}+\mathcal{O}\left(q^{11}\right), \\
& \chi_{a=2}^{(2,7)}(q, T)=\sum_{N_{1} \geqslant N_{2} \geqslant 0} \frac{q^{N_{1}^{2}+N_{2}^{2}+N_{2}}}{(q)_{N_{1}-N_{2}}(q)_{N_{2}}} T^{N_{1}+N_{2}} \\
& =1+T q+T q^{2}+\left(T+T^{2}\right) q^{3}+\left(T+2 T^{2}\right) q^{4}+\left(T+2 T^{2}\right) q^{5} \\
& +\left(T+3 T^{2}+T^{3}\right) q^{6}+\left(T+3 T^{2}+2 T^{3}\right) q^{7}+\left(T+4 T^{2}+3 T^{3}\right) q^{8} \\
& +\left(T+4 T^{2}+5 T^{3}\right) q^{9}+\left(T+5 T^{2}+6 T^{3}+T^{4}\right) q^{10}+\mathcal{O}\left(q^{11}\right), \\
& \chi_{a=1}^{(2,9)}(q, T)=\sum_{N_{1} \geqslant N_{2} \geqslant N_{3} \geqslant 0} \frac{q^{N_{1}^{2}+N_{2}^{2}+N_{3}^{2}+N_{1}+N_{2}+N_{3}}}{(q)_{N_{1}-N_{2}}(q)_{N_{2}-N_{3}}(q)_{N_{3}}} T^{N_{1}+N_{2}+N_{3}} \\
& =1+T q^{2}+T q^{3}+\left(T+T^{2}\right) q^{4}+\left(T+T^{2}\right) q^{5} \\
& +\left(T+2 T^{2}+T^{3}\right) q^{6}+\left(T+2 T^{2}+T^{3}\right) q^{7}+\left(T+3 T^{2}+2 T^{3}\right) q^{8} \\
& +\left(T+3 T^{2}+3 T^{3}\right) q^{9}+\left(T+4 T^{2}+4 T^{3}+T^{4}\right) q^{10}+\mathcal{O}\left(q^{11}\right), \\
& \chi_{a=2}^{(2,9)}(q, T)=\sum_{N_{1} \geqslant N_{2} \geqslant N_{3} \geqslant 0} \frac{q^{N_{1}^{2}+N_{2}^{2}+N_{3}^{2}+N_{2}+N_{3}}}{(q)_{N_{1}-N_{2}}(q)_{N_{2}-N_{3}}(q)_{N_{3}}} T^{N_{1}+N_{2}+N_{3}} \\
& =1+T q+T q^{2}+\left(T+T^{2}\right) q^{3}+\left(T+2 T^{2}\right) q^{4}+\left(T+2 T^{2}+T^{3}\right) q^{5} \\
& +\left(T+3 T^{2}+2 T^{3}\right) q^{6}+\left(T+3 T^{2}+3 T^{3}\right) q^{7}+\left(T+4 T^{2}+4 T^{3}+T^{4}\right) q^{8} \\
& +\left(T+4 T^{2}+6 T^{3}+2 T^{4}\right) q^{9}+\left(T+5 T^{2}+7 T^{3}+4 T^{4}\right) q^{10}+\mathcal{O}\left(q^{11}\right) .
\end{aligned}
$$

We will see later, while comparing with the Macdonald indices computed from the TQFT picture of gauge theories, that they agree with the above expressions (4.6)-(4.11). 


\subsection{The sum expressions of the $\mathcal{W}_{3}$ characters}

The sum expressions for $\left(p, p^{\prime}\right)=(3,7) \mathcal{W}_{3}$ minimal models are developed in [38], and take the form

$$
\begin{aligned}
\chi_{\left(s_{1}, s_{2}\right)=(1,1)}^{(3,7)}(q) & =\sum_{n_{1}, n_{2}, n_{3}, n_{4} \geqslant 0} \frac{q^{\left(n_{1}+n_{2}+n_{3}\right)^{2}+\left(n_{2}+n_{3}\right)^{2}+n_{3}{ }^{2}+n_{4}{ }^{2}+\left(n_{1}+2 n_{2}+3 n_{3}\right) n_{4}+\left(n_{1}+2 n_{2}+3 n_{3}+2 n_{4}\right)}}{(q)_{n_{1}}(q)_{n_{2}}(q)_{n_{3}}(q)_{n_{4}}} \\
& =\frac{1}{\left(q^{2} ; q^{7}\right)\left(q^{3} ; q^{7}\right)^{2}\left(q^{4} ; q^{7}\right)^{2}\left(q^{5} ; q^{7}\right)} \\
\chi_{\left(s_{1}, s_{2}\right)=(1,3)}^{(3,7)}(q) & =\sum_{n_{1}, n_{2}, n_{3}, n_{4} \geqslant 0} \frac{q^{\left(n_{1}+n_{2}+n_{3}\right)^{2}+\left(n_{2}+n_{3}\right)^{2}+n_{3}^{2}+n_{4}^{2}+\left(n_{1}+2 n_{2}+3 n_{3}\right) n_{4}+n_{3}+n_{4}}}{(q)_{n_{1}}(q)_{n_{2}}(q)_{n_{3}}(q)_{n_{4}}} \\
& =\frac{1}{\left(q^{1} ; q^{7}\right)\left(q^{2} ; q^{7}\right)^{2}\left(q^{5} ; q^{7}\right)^{2}\left(q^{6} ; q^{7}\right)}, \\
\chi_{\left(s_{1}, s_{2}\right)=(2,2)}^{(3,7)}(q) & =\sum_{n_{1}, n_{2}, n_{3}, n_{4} \geqslant 0} \frac{q^{\left(n_{1}+n_{2}+n_{3}\right)^{2}+\left(n_{2}+n_{3}\right)^{2}+n_{3}^{2}+n_{4}^{2}+\left(n_{1}+2 n_{2}+3 n_{3}\right) n_{4}}}{(q)_{n_{1}}(q)_{n_{2}}(q)_{n_{3}}(q)_{n_{4}}} \\
& =\frac{1}{\left(q^{1} ; q^{7}\right)^{2}\left(q^{3} ; q^{7}\right)\left(q^{4} ; q^{7}\right)\left(q^{6} ; q^{7}\right)^{2}}, \\
\chi_{\left(s_{1}, s_{2}\right)=(1,2)}^{(3,7)}(q) & =\sum_{n_{1}, n_{2}, n_{3}, n_{4} \geqslant 0} \frac{q^{\left(n_{1}+n_{2}+n_{3}\right)^{2}+\left(n_{2}+n_{3}\right)^{2}+n_{3}^{2}+n_{4}^{2}+\left(n_{1}+2 n_{2}+3 n_{3}\right) n_{4}+\left(n_{2}+2 n_{3}+n_{4}\right)}}{(q)_{n_{1}}(q)_{n_{2}}(q) n_{3}(q)_{n_{4}}} \\
& =\frac{1}{\left(q^{1} ; q^{7}\right)\left(q^{2}\left(q^{1} ; q^{7}\right) ; q^{7}\right)\left(q^{3} ; q^{7}\right)\left(q^{5} ; q^{7}\right)\left(q^{1} ; q^{7}\right)\left(q^{6} ; q^{7}\right)} .
\end{aligned}
$$

The vacuum character (labeled by $s_{1}=s_{2}=1$ ) is given in equation (4.12a) and the character of the next-to-vacuum module, labeled by $s_{1}=1$ and $s_{2}=2$, is given in (4.12d). These two characters will be the main focus of ours in this model.

We remark that as before, $n_{1,2,3,4}$ is the total number of particles of different species. This means that there are four types of fundamental particles in the $\mathcal{W}_{3},\left(p, p^{\prime}\right)=(3,7)$, model, and all states are composition of these fundamental particles or their (Schur) descendants following some selection rules. For example we can write the explicit form of the vacuum character,

$$
\begin{aligned}
\chi_{\left(s_{1}, s_{2}\right)=(1,1)}^{(3,7)}(q)= & +\frac{q^{2}}{1-q}+\frac{q^{3}}{1-q}+\frac{q^{4}}{1-q}+\frac{q^{6}}{1-q}+\frac{q^{6}}{(1-q)^{2}}+\frac{q^{6}}{(1-q)(1-q)^{2}} \\
& +\frac{q^{8}}{(1-q)(1-q)^{2}}+\frac{q^{9}}{(1-q)^{2}}+\frac{q^{10}}{(1-q)(1-q)^{2}}+\cdots
\end{aligned}
$$

where the second term and the third term respectively correspond to $n_{1}=1$ and $n_{4}=1$ (other $n_{i}$ 's being zero), and the sixth term $\frac{q^{6}}{(1-q)^{2}}$ is generated from $n_{1}=n_{4}=1, n_{2}=$ $n_{3}=0$, that is the lowest contribution comes from the composition of a weight 2 particle (counted by $n_{1}$ ) and a weight 4 particle (counted by $n_{4}$ ).

\subsection{The Macdonald version of the sum expressions of the $\mathcal{W}_{3}$ characters}

Now we consider the $T$-refinement of the fermionic characters (4.12a)-(4.12d). As the path picture is currently not completely clear for higher-rank minimal models, the most natural 
generalization for $\mathcal{W}_{3}$ is to add a refinement weight

$$
T^{n_{1}+2 n_{2}+3 n_{3}+2 n_{4}}
$$

to each term in the summation, where $n_{1}+2 n_{2}+3 n_{3}+2 n_{4}$ is the linear term appearing in the power of $q$ in the vacuum character as in the case of $T$-refinement of Virasoro characters. In terms of particles, the refinement weight (4.14) means that we assign a weight $T$ to the first type counted by $n_{1}, T^{2}$ to the second type of particles counted by $n_{2}$ and so on. The refined expressions for each module in $\left(p, p^{\prime}\right)=(3,7)$ model are given below, together with their series expansions in $q$.

$$
\begin{aligned}
\chi_{\left(s_{1}, s_{2}\right)}^{(3,7)}=(1,1) & (q, T) \\
= & \sum_{n_{1}, n_{2}, n_{3}, n_{4} \geqslant 0} \frac{q^{\left(n_{1}+n_{2}+n_{3}\right)^{2}+\left(n_{2}+n_{3}\right)^{2}+n_{3}{ }^{2}+n_{4}{ }^{2}+\left(n_{1}+2 n_{2}+3 n_{3}\right) n_{4}+\left(n_{1}+2 n_{2}+3 n_{3}+2 n_{4}\right)}}{(q)_{n_{1}}(q)_{n_{2}}(q)_{n_{3}}(q)_{n_{4}}} T^{n_{1}+2 n_{2}+3 n_{3}+2 n_{4}} \\
= & +T q^{2}+\left(T+T^{2}\right) q^{3}+\left(T+2 T^{2}\right) q^{4}+\left(T+2 T^{2}\right) q^{5}+\left(T+3 T^{2}+2 T^{3}\right) q^{6} \\
& +\left(T+3 T^{2}+3 T^{3}\right) q^{7}+\left(T+4 T^{2}+5 T^{3}+T^{4}\right) q^{8} \\
& +\left(T+4 T^{2}+7 T^{3}+2 T^{4}\right) q^{9}+\mathcal{O}\left(q^{10}\right)
\end{aligned}
$$

$$
\begin{aligned}
\chi_{\left(s_{1}, s_{2}\right)}^{(3,7)}=(1,3) & (q, T) \\
= & \sum_{n_{1}, n_{2}, n_{3}, n_{4} \geqslant 0} \frac{q^{\left(n_{1}+n_{2}+n_{3}\right)^{2}+\left(n_{2}+n_{3}\right)^{2}+n_{3}^{2}+n_{4}^{2}+\left(n_{1}+2 n_{2}+3 n_{3}\right) n_{4}+n_{3}+n_{4}}}{(q)_{n_{1}}(q)_{n_{2}}(q)_{n_{3}}(q)_{n_{4}}} T^{n_{1}+2 n_{2}+3 n_{3}+2 n_{4}} \\
= & +T q+\left(T+2 T^{2}\right) q^{2}+\left(T+2 T^{2}\right) q^{3}+\left(T+3 T^{2}+2 T^{3}\right) q^{4} \\
& +\left(T+3 T^{2}+4 T^{3}\right) q^{5}+\left(T+4 T^{2}+6 T^{3}+2 T^{4}\right) q^{6} \\
& +\left(T+4 T^{2}+8 T^{3}+4 T^{4}\right) q^{7}+\left(T+5 T^{2}+10 T^{3}+9 T^{4}\right) q^{8} \\
& +\left(T+5 T^{2}+13 T^{3}+12 T^{4}+2 T^{5}\right) q^{9}+\mathcal{O}\left(q^{10}\right)
\end{aligned}
$$

$$
\begin{aligned}
\chi_{\left(s_{1}, s_{2}\right)}^{(3,7)}=(2,2) & (q, T) \\
= & \sum_{n_{1}, n_{2}, n_{3}, n_{4} \geqslant 0} \frac{q^{\left(n_{1}+n_{2}+n_{3}\right)^{2}+\left(n_{2}+n_{3}\right)^{2}+n_{3}^{2}+n_{4}^{2}+\left(n_{1}+2 n_{2}+3 n_{3}\right) n_{4}}}{(q)_{n_{1}}(q)_{n_{2}}(q)_{n_{3}}(q)_{n_{4}}} T^{n_{1}+2 n_{2}+3 n_{3}+2 n_{4}} \\
= & +\left(T+T^{2}\right) q+\left(T+2 T^{2}\right) q^{2}+\left(T+2 T^{2}+2 T^{3}\right) q^{3}+\left(T+3 T^{2}+3 T^{3}+T^{4}\right) q^{4} \\
& +\left(T+3 T^{2}+5 T^{3}+2 T^{4}\right) q^{5}+\left(T+4 T^{2}+7 T^{3}+5 T^{4}\right) q^{6} \\
& +\left(T+4 T^{2}+9 T^{3}+8 T^{4}+2 T^{5}\right) q^{7}+\left(T+5 T^{2}+11 T^{3}+13 T^{4}+4 T^{5}\right) q^{8} \\
& \quad+\left(T+5 T^{2}+14 T^{3}+17 T^{4}+9 T^{5}+T^{6}\right) q^{9}+\mathcal{O}\left(q^{10}\right)
\end{aligned}
$$

$$
\begin{aligned}
\chi_{\left(s_{1}, s_{2}\right)}^{(3,7)} & =(1,2) \\
= & \sum_{n_{1}, n_{2}, n_{3}, n_{4} \geqslant 0} \frac{q^{\left(n_{1}+n_{2}+n_{3}\right)^{2}+\left(n_{2}+n_{3}\right)^{2}+n_{3}^{2}+n_{4}^{2}+\left(n_{1}+2 n_{2}+3 n_{3}\right) n_{4}+\left(n_{2}+2 n_{3}+n_{4}\right)}}{(q)_{n_{1}}(q)_{n_{2}}(q)_{n_{3}}(q)_{n_{4}}} T^{n_{1}+2 n_{2}+3 n_{3}+2 n_{4}} \\
= & 1+T q+\left(T+T^{2}\right) q^{2}+\left(T+2 T^{2}\right) q^{3}+\left(T+3 T^{2}+T^{3}\right) q^{4} \\
& +\left(T+3 T^{2}+3 T^{3}\right) q^{5}+\left(T+4 T^{2}+5 T^{3}+T^{4}\right) q^{6} \\
& +\left(T+4 T^{2}+7 T^{3}+2 T^{4}\right) q^{7}+\left(T+5 T^{2}+9 T^{3}+6 T^{4}\right) q^{8} \\
& \quad+\left(T+5 T^{2}+12 T^{3}+9 T^{4}+T^{5}\right) q^{9}+\mathcal{O}\left(q^{10}\right)
\end{aligned}
$$




\subsection{The ASW sum expressions of $\mathcal{W}_{3}$ characters}

Another version of the sum expressions of the $\mathcal{W}_{3},\left(p, p^{\prime}\right)=(3,7)$, characters is proposed in [8],

$$
\begin{aligned}
& \chi_{\left(s_{1}, s_{2}\right)=(1,1)}^{(3,7)}(q)=\sum_{n_{1}, n_{2} \geqslant 0} \frac{q^{n_{1}^{2}-n_{1} n_{2}+n_{2}^{2}+n_{1}+n_{2}}}{(q)_{n_{1}}}\left[\begin{array}{c}
2 n_{1} \\
n_{2}
\end{array}\right]_{q}, \\
& \chi_{\left(s_{1}, s_{2}\right)=(1,3)}^{(3,7)}(q)=\sum_{n_{1}, n_{2} \geqslant 0} \frac{q^{n_{1}^{2}-n_{1} n_{2}+n_{2}^{2}+n_{2}}}{(q)_{n_{1}}}\left[\begin{array}{c}
2 n_{1}+1 \\
n_{2}
\end{array}\right]_{q} \\
& \chi_{\left(s_{1}, s_{2}\right)=(2,2)}^{(3,7)}(q)=\sum_{n_{1}, n_{2} \geqslant 0} \frac{q^{n_{1}^{2}-n_{1} n_{2}+n_{2}^{2}}}{(q)_{n_{1}}}\left[\begin{array}{c}
2 n_{1} \\
n_{2}
\end{array}\right]_{q}, \\
& \chi_{\left(s_{1}, s_{2}\right)=(1,2)}^{(3,7)}(q)=\sum_{n_{1}, n_{2} \geqslant 0} \frac{q^{n_{1}^{2}-n_{1} n_{2}+n_{2}^{2}+n_{1}}}{(q)_{n_{1}}}\left[\begin{array}{c}
2 n_{1}+1 \\
n_{2}
\end{array}\right]_{q}=\sum_{n_{1}, n_{2} \geqslant 0} \frac{q^{n_{1}^{2}-n_{1} n_{2}+n_{2}^{2}+n_{2}}}{(q)_{n_{1}}}\left[\begin{array}{c}
2 n_{1} \\
n_{2}
\end{array}\right]_{q},
\end{aligned}
$$

where

is the $q$-Gaussian polynomial. ${ }^{15}$

$$
\left[\begin{array}{l}
P \\
N
\end{array}\right]_{q}= \begin{cases}\frac{(q)_{P}}{(q)_{N}(q)_{P-N}} & 0 \leqslant N \leqslant P \\
0 & \text { otherwise }\end{cases}
$$

As before, we wish to refine the characters (4.16a)-(4.16d) with $T$ to the power of the linear term in the power of $q$ in the numerator of the vacuum character, that is, $T^{n_{1}+n_{2}}$. The Macdonald version of the $\mathcal{W}_{3}$ characters that we obtain in this way are

$$
\begin{aligned}
\chi_{\left(s_{1}, s_{2}\right)}^{(3,7)}=(1,1) & (q, T) \\
= & \sum_{n_{1}, n_{2} \geqslant 0} \frac{q^{n_{1}^{2}-n_{1} n_{2}+n_{2}^{2}+n_{1}+n_{2}}}{(q)_{n_{1}}}\left[\begin{array}{c}
2 n_{1} \\
n_{2}
\end{array}\right]_{q} T^{n_{1}+n_{2}} \\
= & +T q^{2}+\left(T+T^{2}\right) q^{3}+\left(T+2 T^{2}\right) q^{4}+\left(T+2 T^{2}\right) q^{5}+\left(T+3 T^{2}+2 T^{3}\right) q^{6} \\
& +\left(T+3 T^{2}+3 T^{3}\right) q^{7}+\left(T+4 T^{2}+5 T^{3}+T^{4}\right) q^{8} \\
& +\left(T+4 T^{2}+7 T^{3}+2 T^{4}\right) q^{9}+\mathcal{O}\left(q^{10}\right), \\
\chi_{\left(s_{1}, s_{2}\right)}^{(3,7)}=(1,3) & (q, T) \\
= & \sum_{n_{1}, n_{2} \geqslant 0} \frac{q^{n_{1}^{2}-n_{1} n_{2}+n_{2}^{2}+n_{2}}}{(q)_{n_{1}}}\left[\begin{array}{c}
2 n_{1}+1 \\
n_{2}
\end{array}\right]_{q} T^{n_{1}+n_{2}} \\
= & +T q+(1+2 T) q^{2}+3 T q^{3}+\left(4 T+2 T^{2}\right) q^{4}+\left(5 T+3 T^{2}\right) q^{5}+\left(6 T+7 T^{2}\right) q^{6} \\
& +\left(7 T+10 T^{2}\right) q^{7}+\left(7 T+17 T^{2}+T^{3}\right) q^{8}+\left(7 T+22 T^{2}+4 T^{3}\right) q^{9}+\mathcal{O}\left(q^{10}\right),
\end{aligned}
$$

$$
\begin{aligned}
& \chi_{\left(s_{1}, s_{2}\right)=(2,2)}^{(3,7)}(q, T) \\
& \quad=\sum_{n_{1}, n_{2} \geqslant 0} \frac{q^{n_{1}^{2}-n_{1} n_{2}+n_{2}^{2}}}{(q)_{n_{1}}}\left[\begin{array}{c}
2 n_{1} \\
n_{2}
\end{array}\right]_{q} T^{n_{1}+n_{2}}
\end{aligned}
$$

\footnotetext{
${ }^{15}$ Equation (4.16b) has long time been a conjectured expression but was proved in [32] recently.
} 


$$
\begin{aligned}
= & +\left(T+T^{2}\right) q+\left(T+2 T^{2}\right) q^{2}+\left(T+2 T^{2}+2 T^{3}\right) q^{3}+\left(T+3 T^{2}+3 T^{3}+T^{4}\right) q^{4} \\
& +\left(T+3 T^{2}+5 T^{3}+2 T^{4}\right) q^{5}+\left(T+4 T^{2}+7 T^{3}+5 T^{4}\right) q^{6} \\
& +\left(T+4 T^{2}+9 T^{3}+8 T^{4}+2 T^{5}\right) q^{7}+\left(T+5 T^{2}+11 T^{3}+13 T^{4}+4 T^{5}\right) q^{8} \\
& +\left(T+5 T^{2}+14 T^{3}+17 T^{4}+9 T^{5}+T^{6}\right) q^{9}+\mathcal{O}\left(q^{10}\right),
\end{aligned}
$$

$$
\begin{aligned}
\chi_{\left(s_{1}, s_{2}\right)}^{(3,7)}=(1,2) & (q) \\
= & \sum_{n_{1}, n_{2} \geqslant 0} \frac{q^{n_{1}^{2}-n_{1} n_{2}+n_{2}^{2}+n_{1}}}{(q)_{n_{1}}}\left[\begin{array}{c}
2 n_{1}+1 \\
n_{2}
\end{array}\right]_{q} T^{n_{1}+n_{2}} \\
= & \sum_{n_{1}, n_{2} \geqslant 0} \frac{q^{n_{1}^{2}-n_{1} n_{2}+n_{2}^{2}+n_{2}}}{(q)_{n_{1}}}\left[\begin{array}{c}
2 n_{1} \\
n_{2}
\end{array}\right]_{q} T^{n_{1}+n_{2}} \\
= & +T q+\left(T+T^{2}\right) q^{2}+\left(T+2 T^{2}\right) q^{3}+\left(T+3 T^{2}+T^{3}\right) q^{4} \\
& +\left(T+3 T^{2}+3 T^{3}\right) q^{5}+\left(T+4 T^{2}+5 T^{3}+T^{4}\right) q^{6}+\left(T+4 T^{2}+7 T^{3}+2 T^{4}\right) q^{7} \\
& +\left(T+5 T^{2}+9 T^{3}+6 T^{4}\right) q^{8}+\left(T+5 T^{2}+12 T^{3}+9 T^{4}+T^{5}\right) q^{9}+\mathcal{O}\left(q^{10}\right)
\end{aligned}
$$

We observe that $(4.18 \mathrm{a}),(4.18 \mathrm{c})$ and $(4.18 \mathrm{~d})$ respectively match $(4.15 \mathrm{a}),(4.15 \mathrm{c})$ and $(4.15 \mathrm{~d})$ as series expansions, while (4.18b) does not match (4.15b). Since we only consider vacuum and next-to-vacuum characters, (4.15a) and (4.15d), in this article, this disagreement is not important to us at the moment.

\subsection{Matching the Virasoro infinite-series of vacuum characters}

Let us list the Macdonald indices obtained in [75, 76, 85].

$$
\begin{aligned}
\mathcal{I}^{\left(A_{1}, A_{2}\right)}=1 & +T q^{2}+T q^{3}+T q^{4}+T q^{5}+\left(T+T^{2}\right) q^{6}+\left(T+T^{2}\right) q^{7}+\left(T+2 T^{2}\right) q^{8} \\
& +\left(T+2 T^{2}\right) q^{9}+\left(T+3 T^{2}\right) q^{10}+\mathcal{O}\left(q^{11}\right) \\
\mathcal{I}^{\left(A_{1}, A_{4}\right)}=1 & +T q^{2}+T q^{3}+\left(T+T^{2}\right) q^{4}+\left(T+T^{2}\right) q^{5}+\left(T+2 T^{2}\right) q^{6} \\
& +\left(T+2 T^{2}\right) q^{7}+\left(T+3 T^{2}+T^{3}\right) q^{8}+\left(T+3 T^{2}+2 T^{3}\right) q^{9} \\
& +\left(T+4 T^{2}+3 T^{3}\right) q^{10}+\mathcal{O}\left(q^{11}\right) \\
\mathcal{I}^{\left(A_{1}, A_{6}\right)}= & +T q^{2}+T q^{3}+\left(T+T^{2}\right) q^{4}+\left(T+T^{2}\right) q^{5}+\left(T+2 T^{2}+T^{3}\right) q^{6} \\
& +\left(T+2 T^{2}+T^{3}\right) q^{7}+\left(T+3 T^{2}+2 T^{3}\right) q^{8}+\left(T+3 T^{2}+3 T^{3}\right) q^{9} \\
& +\left(T+4 T^{2}+4 T^{3}+T^{4}\right) q^{10}+\mathcal{O}\left(q^{11}\right)
\end{aligned}
$$

The above results (4.19), (4.20) and (4.21) match the $t$-refined characters obtained from our path approach (4.6), (4.8) and (4.10).

\subsection{Matching the Virasoro infinite-series of next-to-vacuum characters}

Following [85], the Macdonald indices corresponding to the next-to-vacuum modules, computed by inserting a surface defect with vortex number $s^{\prime}=1$, are

$$
\begin{aligned}
\mathcal{I}_{\left(A_{1}, A_{2}\right)}^{\mathbb{S}^{1}}(q, t)= & +T q+T q^{2}+T q^{3}+\left(T+T^{2}\right) q^{4}+\left(T+T^{2}\right) q^{5}+\left(T+2 T^{2}\right) q^{6} \\
& +\left(T+2 T^{2}\right) q^{7}+\left(T+3 T^{2}\right) q^{8}+\left(T+3 T^{2}+T^{3}\right) q^{9} \\
& +\left(T+4 T^{2}+T^{3}\right) q^{10}+\mathcal{O}\left(q^{11}\right),
\end{aligned}
$$




$$
\begin{aligned}
\mathcal{I}_{\left(A_{1}, A_{4}\right)}^{\mathbb{S}^{1}}(q, t)= & +T q+T q^{2}+\left(T+T^{2}\right) q^{3}+\left(T+2 T^{2}\right) q^{4}+\left(T+2 T^{2}\right) q^{5} \\
& +\left(T+3 T^{2}+T^{3}\right) q^{6}+\left(T+3 T^{2}+2 T^{3}\right) q^{7}+\left(T+4 T^{2}+3 T^{3}\right) q^{8} \\
& +\left(T+4 T^{2}+5 T^{3}\right) q^{9}+\left(T+5 T^{2}+6 T^{3}+T^{4}\right) q^{10}+\mathcal{O}\left(q^{11}\right), \quad\left(T+2 T^{2}\right) q^{4}+\left(T+2 T^{2}+T^{3}\right) q^{5} \\
\mathcal{I}_{\left(A_{1}, A_{6}\right)}^{\mathbb{S}^{1}}(q, t)= & +T q+T q^{2}+\left(T+T^{2}\right) q^{3}+\left(T+2 T^{2}\right) q^{7} \\
& +\left(T+3 T^{2}+2 T^{3}\right) q^{6}+\left(T+3 T^{2}+3 T^{3}\right) q^{9} \\
& +\left(T+4 T^{2}+4 T^{3}+T^{4}\right) q^{8}+\left(T+4 T^{2}+6 T^{3}+2 T^{3}\right) q^{10}+\mathcal{O}\left(q^{11}\right)
\end{aligned}
$$

and they match (4.7), (4.9) and (4.11) computed from the path approach.

\subsection{Matching the $\mathcal{W}_{3}$ vacuum and next-to-vacuum characters}

The Macdonald indices for rank-two Argyres-Douglas theories are also computed in [85] via the TQFT approach, and the indices corresponding to the next-to-vacuum module are also conjectured based on the Higgsing approach. In this way, we obtained

$$
\begin{aligned}
\mathcal{I}^{\left(A_{2}, A_{3}\right)}(q, t)= & +T q^{2}+\left(T+T^{2}\right) q^{3}+\left(T+2 T^{2}\right) q^{4}+\left(T+2 T^{2}\right) q^{5} \\
& +\left(T+3 T^{2}+2 T^{3}\right) q^{6}+\left(T+3 T^{2}+3 T^{3}\right) q^{7}+\left(T+4 T^{2}+5 T^{3}+T^{4}\right) q^{8} \\
& +\left(T+4 T^{2}+7 T^{3}+2 T^{4}\right) q^{9}+\mathcal{O}\left(q^{10}\right), \\
\mathcal{I}_{\left(A_{2}, A_{3}\right)}^{\mathbb{S}^{1,0}}(q, t)= & +T q+\left(T+T^{2}\right) q^{2}+\left(T+2 T^{2}\right) q^{3}+\left(T+3 T^{2}+T^{3}\right) q^{4} \\
& +\left(T+3 T^{2}+3 T^{3}\right) q^{5}+\left(T+4 T^{2}+5 T^{3}+T^{4}\right) q^{6} \\
& +\left(T+4 T^{2}+7 T^{3}+2 T^{4}\right) q^{7}+\mathcal{O}\left(q^{8}\right)
\end{aligned}
$$

Interestingly, (4.25) and (4.26) respectively match with (4.15a) and (4.15d) (or equivalently (4.18a) and (4.18d)) up to the order computed for the Macdonald index.

Remark. The above indices (4.25) and (4.26) are computed in the TQFT appraoch only with the wavefunction $f_{\varnothing}^{I_{3,4}}(q, t)$ and $f_{(2,1)}^{I_{3,4}}(q, t)$, and are truncated at the level that is not affected by the next non-trivial contributions from $f_{(3,0)}^{I_{3,4}}(q, t)$ and $f_{(3,3)}^{I_{3,4}}(q, t)$.

\subsection{Relation with Schur operators}

Here, we focus on the cases corresponding to Virasoro minimal models, where the paths picture is well-understood. For the Lee-Yang model $\mathcal{L}^{2,5}$, the vacuum character is

$$
\sum_{N_{1} \geqslant 0} \frac{q^{N_{1}^{2}+N_{1}}}{(q)_{N_{1}}}=\sum_{N_{1} \geqslant 0} \sum_{\substack{t_{1}, t_{2}, \cdots, t_{N_{1}} \\ t_{i+1}-t_{i} \geqslant 2, t_{1} \geqslant 2}} q^{\sum_{i=1}^{N_{1}} t_{i}},
$$

and its $t$-refined version is

$$
\sum_{N_{1} \geqslant 0} \frac{q^{N_{1}^{2}+N_{1}}}{(q)_{N_{1}}} T^{N_{1}}=\sum_{N_{1}=0}^{\infty} T^{N_{1}} \sum_{\begin{array}{c}
t_{1}, t_{2}, \cdots, t_{N_{1}} \\
t_{i+1}-t_{i} \geqslant 2 \\
t_{1} \geqslant 2
\end{array}} q^{\sum_{i=1}^{N_{1}} t_{i}}
$$


Let $\mathcal{O}$ denote the primary Schur operator that corresponds to the contribution $T q^{2}$ in the Macdonald index. Each particle with weight $t_{i}$ corresponds to $t_{i}-2$ derivatives ${ }^{16}$ acting on $\mathcal{O}$, that is, the operator $\left(\sigma_{++}^{\mu} \partial_{\mu}\right)^{t_{i}-2} \mathcal{O}$. A general composite Schur operator made from $N_{1}$ such building blocks, of the form : $\prod_{i=1}^{N_{1}}\left(\sigma_{+\dot{+}}^{\mu} \partial_{\mu}\right)^{t_{i}-2} \mathcal{O}:$, then corresponds to a path with $N_{1}$ particles of weight $t_{i}$. It is natural in this context to conjecture that there is only one primary Schur operator, $\mathcal{O}$, in the $\left(A_{1}, A_{2}\right)$ theory. Due to the fermionic nature of the particles, : $\mathcal{O O}$ :, for example, is not allowed in the spectrum. This corresponds to the superselection rule in the OPE of Schur operators.

Similarly, in the $\Delta=-\frac{1}{5}$ module of the Lee-Yang model $\mathcal{L}^{2,5}$, we prepare an operator $\mathcal{J}$ that corresponds to the contribution $T q$ in the Macdonald index, then all peaks and valleys in the statistical mechanical model (with weight $t_{i}$ ) correspond to a Schur operator $\left(\sigma_{+\dot{+}}^{\mu} \partial_{\mu}\right)^{t_{i}-1} \mathcal{J}$. Each path with several peaks and valleys represents a composite Schur operator as a product : $\prod_{i}\left(\sigma_{+\dot{+}}^{\mu} \partial_{\mu}\right)^{t_{i}-1} \mathcal{J}$ :

The case of $\mathcal{L}^{2,7}$ model is more interesting. In the vacuum module, we have two types of particles when the weight is larger than or equal to 4 . At level 4 , we have a descendant Schur operator $\left(\sigma_{+\dot{+}}^{\mu} \partial_{\mu}\right)^{2} \mathcal{O}$, which contributes $T q^{4}$ to the Macdonald index, and a primary Schur operator $\hat{\mathcal{C}}_{1\left(\frac{1}{2}, \frac{1}{2}\right)} \sim: \mathcal{O O}$ :, which has Macdonald weight $T^{2} q^{4}$. The contribution from $\hat{\mathcal{C}}_{2(1,1)} \sim: \mathcal{O O O}$ : is missing in the Macdonald index, which agrees with the argument for the vanishing of the OPE coefficient $\lambda\left[\mathcal{O}, \hat{\mathcal{C}}_{1\left(\frac{1}{2}, \frac{1}{2}\right)}, \hat{\mathcal{C}}_{2(1,1}\right]$ in [2]. This superselection rules is easily understood in the language of paths.

More generally, the vanishing of the OPE coefficient $\lambda\left[\mathcal{O}, \hat{\mathcal{C}}_{k\left(\frac{k}{2}, \frac{k}{2}\right)}, \hat{\mathcal{C}}_{(k+1)\left(\frac{k+1}{2}, \frac{k+2}{2}\right.}\right]$ matches with the fact that there are only $k$ types of particles in the statistical mechanical model of paths, and supports our conjecture regarding the correspondence between the Schur operators and the paths.

In the case of $\left(p, p^{\prime}\right)=(3,7)$, there are four types of particles in the fermionic sums (4.12a) to (4.12d). From the discussion of [2] to the effect that $\mathcal{W}^{2}$ is not included in the spectrum, where $\mathcal{W}=\mathcal{C}_{1(0,0)}$, etc., it is consistent to identify the four primary Schur operators as $\mathcal{O}=\hat{\mathcal{C}}_{0(0,0)}, \hat{\mathcal{C}}_{1\left(\frac{1}{2}, \frac{1}{2}\right)} \sim: \mathcal{O}^{2}:, \hat{\mathcal{C}}_{2(1,1)} \sim: \mathcal{O}^{3}:$, and $\mathcal{W}=\mathcal{C}_{1(0,0)}$, whose refinement weights are respectively $T, T^{2}, T^{3}$ and $T^{2}$. In particular, the weight $T^{2}$ for $\mathcal{W}$ agrees with the prescription given in [85]. The consistency with previous works on the gauge theory side also suggests that the formulation of (4.12a) to (4.12d) is essentially a free theory approach.

\section{Comments}

\subsection{Surface operators and characters}

Only the Macdonald indices computed in [85] that correspond to the vacuum module or the next-to-vacuum module (that is, in the Virasoro case, the $(r=1, s=1)$ and $(r=1, s=2)$

\footnotetext{
${ }^{16} \sigma_{\alpha \dot{\alpha}}^{\mu}$ or more explicitly $\left(\sigma^{\mu}\right)_{\mu=0}^{3}=\left(\mathbf{1}, \sigma^{1}, \sigma^{2}, \sigma^{3}\right)$ is the a collection of Pauli matrices that can be used to convert the representation of the $\mathrm{SO}(4)$ Lorentz group to the spinors of $\mathrm{SU}(2) \times \mathrm{SU}(2) . \sigma_{++}^{\mu}$ is the top component of this matrix, as a Schur operator always has to be the highest-weight state in the representation of Lorentz group [10].
} 
modules, and in the $\mathcal{W}_{3}$ case, the $\left(r_{1}, r_{2}, s_{1}, s_{2}\right)=(1,1,1,1)$ and $\left(r_{1}, r_{2}, s_{1}, s_{2}\right)=(1,1,1,2)$ modules), are observed to directly take the form of a $t$-refined character. The Macdonald indices for more complicated modules, obtained using the same method, contain negative contributions. It is not clear whether only the Macdonald indices of the vacuum and the next-to-vacuum module have a physical meaning as $t$-refined characters in the dual chiral algebra.

\subsection{Refining the bosonic version of a character}

In the case of Virasoro characters, it is possible to $t$-refine the bosonic version of a character using the Bailey lattice method of [3]. ${ }^{17}$ However, The Bailey refinement is a complicated one, as it involves not just the parameter $t$, but also the Bailey sequences $\alpha_{n}$ and $\beta_{n}$, $n=0,1, \cdots$. The $\beta$ sequence can be trivialized $\left(\beta_{n}=\frac{1}{(q)_{n}}, n=0,1, \cdots\right)$ to obtain the refined fermionic version that we want (so we know that this is the correct $t$-refinement, but the bosonic version will now involve the $\alpha_{n}$ sequence and becomes quite complicated. For that reason, it seems to us that there is no advantage to $t$-refining the bosonic version in the case of Virasoro characters, since we know the $t$-refined fermionic versions, and we expect that the situation can get only (much) more complicated in the case of $\mathcal{W}_{3}$ algebras where very little, and more general $W_{N}$ algebras where nothing is known about the fermionic versions of the characters or the Bailey lattice.

\subsection{The works of Bourdier, Drukker and Felix}

In [20, 21], Bourdier, Drukker and Felix observed that the Schur index of certain theories can be written in terms of the partition function of a gas of fermions on a circle. It is not clear to us at this stage whether the latter fermions are related to ours. However, it is also entirely possible that the results of $[20,21]$ can be t-refined to obtain Macdonald indices. Further discussion of this is beyond the scope of this work.

\subsection{The works of Beem, Bonetti, Meneghelli, Peelaers and Rastelli}

Our work is definitely restricted to Song's approach to the Macdonald indices in $W_{N}$ models. In that approach, Song basically constructs the bosonic version of the character. Moreover, our work is restricted to those characters that we know the fermionic version thereof. It is entirely possible that the approach of the recent works $[12,13,19]$ is the right one to compute the Macdonald index in closed form in all generality.

\subsection{Paths, particles, instantons, BPS states and the Bethe/Gauge correspon- dence}

The paths are combinatorial objects that naturally belong to the representation theory of Virasoro irreducible highest weight modules. ${ }^{18}$ Following McCoy and collaborators [14, 16, $17,57]$ on the fermionic expressions of the Virasoro characters, the paths are interpreted in

\footnotetext{
${ }^{17}$ We thank O Warnaar for bringing this to our attention.

${ }^{18}$ The corresponding objects in the case of $W_{N}$ irreducible highest weight modules are Young tableaux that obey specific conditions [33, 34].
} 
terms of (quasi-)particles and (quasi-)momenta [44-46, 48]. Subsequently, attempts were made to obtain the fermionic expressions of more elaborate objects, such as the correlation functions in statistical mechanics, or the conformal blocks in $2 \mathrm{D}$ conformal field theories without success [63].

After the discovery of Nekrasov's instanton partition function and the AGT correspondence, it became clear from [18] that the fermionic expressions of the 2D conformal blocks in Virasoro minimal models are the Nekrasov instanton partition functions, and that the particles on the statistical mechanics/conformal field theory side are in correspondence with the instantons on the gauge theory side.

What we obtain in this work is a correspondence of a different type: a correspondence between the particles and the BPS states in Argyres-Douglas theories on the gauge side. It is natural to speculate that the Bethe/Gauge correspondence of Nekrasov and Shatashvili $[64,65]$ lies behind the results that we have obtained in this work.

\subsection{The thermodynamic Bethe Ansatz}

Connections between the combinatorics of the thermodynamic Bethe Ansatz and the combinatorics encoded in the paths were made clear in [16], and further in [49, 81, 86]. We anticipate that the methods of the thermodynamic Bethe Ansatz can be used to compute physical quantities in Argyres-Douglas theories.

\section{Acknowledgments}

We thank Jean-Emile Bourgine, Matthew Buican, Dongmin Gang, Ian Grojnowski, Ralph Kaufmann, Hee-Chol Kim, Kimyeong Lee, Wolfger Peelaers, Leonardo Rastelli, Jaewon Song, S Ole Warnaar, Akimi Watanabe and Trevor Welsh for comments, correspondence and discussions. RZ wishes to thank APCTP and KIAS for hospitality, where this work was finalized.

Open Access. This article is distributed under the terms of the Creative Commons Attribution License (CC-BY 4.0), which permits any use, distribution and reproduction in any medium, provided the original author(s) and source are credited.

\section{References}

[1] P. Agarwal, K. Maruyoshi and J. Song, $\mathcal{N}=1$ deformations and $R G$ flows of $\mathcal{N}=2$ SCFTs. Part II. Non-principal deformations, JHEP 12 (2016) 103 [Addendum ibid. 04 (2017) 113] [arXiv: 1610.05311] [INSPIRE].

[2] P. Agarwal, S. Lee and J. Song, Vanishing OPE coefficients in $4 d N=2$ SCFTs, JHEP 06 (2019) 102 [arXiv: 1812.04743] [INSPIRE].

[3] A Agarwal, G E Andrews and D Bressoud, The Bailey lattice, J. Indina Math. Soc. New Ser. 51 (1987) 57.

[4] O Aharony et al., The Hagedorn-deconfinement phase transition in weakly coupled large $N$ gauge theories, Adv. Theor. Math. Phys. 8 (2004) 603 [hep-th/0310285]. 
[5] G.E. Andrews, R.J. Baxter and P.J. Forrester, Eight-vertex SOS model and generalized Rogers-Ramanujan-type identities, J. Stat. Phys. 35 (1984) 193.

[6] G.E. Andrews, An analytic generalization of the Rogers-Ramanujan identities for odd moduli, Proc. Natl. Acad. Sci. U.S.A. 71 (1974) 4082.

[7] G.E. Andrews, Multiple series Rogers-Ramanujan type identities, Pacif. J. Math. 114 (1984) 267.

[8] G.E. Andrews, A. Schilling and S.O. Warnaar, An $A_{2}$ Bailey lemma and Rogers-Ramanujan-type identities, J. Amer. Math. Soc. 12 (1999) 677,

[9] W.N. Bailey, Some identities in combinatory analysis, Proc. London Math. Soc. 49 (1947) 421.

[10] C. Beem et al., Infinite chiral symmetry in four dimensions, Commun. Math. Phys. 336 (2015) 1359 [arXiv: 1312.5344] [INSPIRE].

[11] C. Beem, W. Peelaers, L. Rastelli and B.C. van Rees, Chiral algebras of class S, JHEP 05 (2015) 020 [arXiv: 1408.6522] [INSPIRE].

[12] C. Beem, C. Meneghelli and L. Rastelli, Free field realizations from the Higgs branch, JHEP 09 (2019) 058 [arXiv: 1903.07624] [INSPIRE].

[13] C. Beem, C. Meneghelli, W. Peelaers and L. Rastelli, VOAs and rank-two instanton SCFTs, arXiv: 1907.08629 [INSPIRE].

[14] A. Berkovich, Fermionic counting of RSOS states and Virasoro character formulas for the unitary minimal series $M(\nu, \nu+1)$, Nucl. Phys. B 431 (1994) 315 [hep-th/9403073] [INSPIRE].

[15] A. Berkovich and B.M. McCoy, The Andrews-Gordon identities and $\bar{q}$-multinomial coefficients, Lett. Math. Phys. bf 37 (1996) 49 [q-alg/9601012].

[16] A. Berkovich and B.M. McCoy, Continued fractions and Fermionic representations for characters of $M\left(p, p^{\prime}\right)$ minimal models, Lett. Math. Phys. 37 (1996) 49 [hep-th/9412030] [INSPIRE].

[17] A. Berkovich, B.M. McCoy and A Schilling, Rogers-Schur-Ramanujan type identities for the $M\left(p, p^{\prime}\right)$ minimal models of conformal field theory, Commun. Math. Phys. 191 (1998) 325 [q-alg/9607020].

[18] M. Bershtein and O. Foda, AGT, Burge pairs and minimal models, JHEP 06 (2014) 177 [arXiv: 1404.7075] [INSPIRE].

[19] F. Bonetti, C. Meneghelli and L. Rastelli, VOAs labelled by complex reflection groups and $4 d$ SCFTs, JHEP 05 (2019) 155 [arXiv:1810.03612] [INSPIRE].

[20] J. Bourdier, N. Drukker and J. Felix, The exact Schur index of $\mathcal{N}=4$ SYM, JHEP 11 (2015) 210 [arXiv: 1507.08659] [INSPIRE].

[21] J. Bourdier, N. Drukker and J. Felix, The $\mathcal{N}=2$ Schur index from free fermions, JHEP 01 (2016) 167 [arXiv: 1510.07041] [INSPIRE].

[22] D. Bressoud, Lattice paths and the Rogers-Ramanujan identities, in the Proceedings of the international Ramanujan centenary conference, Madras (1987), K. Alladi ed., Lecture Notes in Mathematics volume 1395, Springer, Germany (1989).

[23] M. Buican and T. Nishinaka, On the superconformal index of Argyres-Douglas theories, $J$. Phys. 49 (2016) 015401 [arXiv: 1505.05884] [INSPIRE]. 
[24] M. Buican and T. Nishinaka, Argyres-Douglas theories, the Macdonald index, and an RG inequality, JHEP 02 (2016) 159,

[25] M. Buican and T. Nishinaka, On irregular singularity wave functions and superconformal indices, JHEP 09 (2017) 066 [arXiv:1705.07173] [INSPIRE].

[26] T. Creutzig, W-algebras for Argyres-Douglas theories, arXiv:1701.05926.

[27] T. Creutzig, Logarithmic W-algebras and Argyres-Douglas theories at higher rank, JHEP 11 (2018) 188 [arXiv: 1809.01725] [INSPIRE].

[28] C. Cordova and S.-H. Shao, Schur indices, BPS particles and Argyres-Douglas theories, JHEP 01 (2016) 040 [arXiv: 1506. 00265] [INSPIRE].

[29] C. Cordova, D. Gaiotto and S.-H. Shao, Infrared computations of defect Schur indices, JHEP 11 (2016) 106 [arXiv:1606.08429] [INSPIRE].

[30] C. Cordova, D. Gaiotto and S.-H. Shao, Surface defect indices and 2d-4d BPS states, JHEP 12 (2017) 078 [arXiv: 1703.02525] [inSPIRE].

[31] C. Cordova, D. Gaiotto and S.-H. Shao, Surface defects and chiral algebras, JHEP 05 (2017) 140 [arXiv: 1704.01955] [INSPIRE].

[32] S. Corteel and T. Welsh, The $A_{2}$ Rogers-Ramanujan identities revisited, arXiv:1905.08343.

[33] S. Dasmahapatra, On the combinatorics of row and corner transfer matrices of the $A_{n-1}^{(1)}$ restricted face models, Int. J. Mod. Phys. A 12 (1997) 3551 [hep-th/9512095] [INSPIRE].

[34] S. Dasmahapatra and O. Foda, Strings, paths, and standard tableaux, Int. J. Mod. Phys. A 13 (1998) 501 [q-alg/9601011].

[35] M. Dedushenko, From VOAs to short star products in SCFT, arXiv:1911.05741 [INSPIRE].

[36] M. Dedushenko and M. Fluder, Chiral algebra, localization, modularity, surface defects, and all that, arXiv:1904.02704 [INSPIRE].

[37] M. Dedushenko and Y. Wang, $4 d / 2 d \rightarrow 3 d / 1 d$ : a song of protected operator algebras, arXiv: 1912.01006 [INSPIRE].

[38] B. Feigin, O. Foda and T. Welsh, Andrews-Gordon identities from combinations of Virasoro characters, Ramanujan J. 17 (2008) 33 [math-ph/0504014] [INSPIRE].

[39] B.L. Feigin and D.B. Fuchs, Verma modules over the Virasoro algebra, Funct. Anal. Appl. 17 (1983) 241.

[40] M. Fluder and J. Song, Four-dimensional lens space index from two-dimensional chiral algebra, JHEP 07 (2018) 073 [arXiv:1710.06029] [INSPIRE].

[41] M. Fluder and P. Longhi, An infrared bootstrap of the Schur index with surface defects, JHEP 09 (2019) 062 [arXiv: 1905.02724] [INSPIRE].

[42] O. Foda, unpublished (2018).

[43] O. Foda, Off-critical local height probabilities on a plane and critical partition functions on a cylinder, arXiv:1711.03337 [INSPIRE].

[44] O. Foda, K.S.M. Lee, Y. Pugai and T.A. Welsh, Path generating transforms, Contemp. Math. 254 (2000) 157 [math/9810043] [INSPIRE].

[45] O. Foda and Y.-H. Quano, Polynomial identities of the Rogers-Ramanujan type, Int. J. Mod. Phys. A 10 (1995) 2291 [hep-th/9407191] [INSPIRE]. 
[46] O. Foda and Y.-H. Quano, Virasoro character identities from the Andrews-Bailey construction, Int. J. Mod. Phys. A 12 (1997) 1651 [hep-th/9408086] [INSPIRE].

[47] O. Foda and S. Warnaar, A bijection which implies Melzer's polynomial identities, Lett. Math. Phys. 36 (1996) 145 [hep-th/9501088] [INSPIRE].

[48] O. Foda and T.A. Welsh, On the combinatorics of Forrester-Baxter models, Phys. Comb. Kyoto Japan (1999) 49.

[49] O. Foda and T. Welsh, unpublished (2002).

[50] P.J. Forrester and R.J. Baxter, Further exact solutions of the eight-vertex SOS model and generalizations of the Rogers-Ramanujan identities, J. Stat. Phys. 38 (1985) 435.

[51] A. Gadde, L. Rastelli, S.S. Razamat and W. Yan, The 4d superconformal index from q-deformed 2d Yang-Mills, Phys. Rev. Lett. 106 (2011) 241602 [arXiv:1104.3850] [InSPIRE].

[52] A. Gadde, L. Rastelli, S.S. Razamat and W. Yan, Gauge theories and Macdonald polynomials, Commun. Math. Phys. 319 (2013) 147 [arXiv:1110.3740] [InSPIRE].

[53] D. Gaiotto, L. Rastelli and S.S. Razamat, Bootstrapping the superconformal index with surface defects, JHEP 01 (2013) 022 [arXiv:1207.3577] [INSPIRE].

[54] G. Gasper and M. Rahman, Basic hypergeometric series, Encyclopedia of Mathematics And its Applications volume 35, Cambridge University Press, Cambridge U.K. (1990).

[55] B. Gordon, A combinatorial generalization of the Rogers-Ramanujan identities, Amer. J. Math. 83 (1961) 393.

[56] R.M. Kaufmann, Path space decompositions for the Virasoro algebra and its Verma modules, Int. J. Mod. Phys. A 10 (1995) 943 [hep-th/9405041] [INSPIRE].

[57] R. Kedem, T.R. Klassen, B.M. McCoy and E. Melzer, Fermionic sum representations for conformal field theory characters, Phys. Lett. B 307 (1993) 68 [hep-th/9301046] [INSPIRE].

[58] J. Kellendonk and A. Recknagel, Virasoro representations on fusion graphs, Phys. Lett. B 298 (1993) 329 [hep-th/9210007] [INSPIRE].

[59] J. Kellendonk, M. Rosgen and R. Varnhagen, Path spaces and $W$ fusion in minimal models, Int. J. Mod. Phys. A 9 (1994) 1009 [hep-th/9301086] [INSPIRE].

[60] J. Kinney, J. Maldacena, S. Minwalla and S. Raju, An index for 4 dimensional super conformal theories, Commun. Math. Phys. 275 (2007) 209 [hep-th/0510251].

[61] K. Maruyoshi and J. Song, Enhancement of supersymmetry via renormalization group flow and the superconformal index, Phys. Rev. Lett. 118 (2017) 151602 [arXiv:1606.05632] [INSPIRE].

[62] K. Maruyoshi and J. Song, $\mathcal{N}=1$ deformations and $R G$ flows of $\mathcal{N}=2$ SCFTs, JHEP 02 (2017) 075 [arXiv: 1607.04281] [INSPIRE].

[63] B.M. McCoy, private communication.

[64] N.A. Nekrasov and S.L. Shatashvili, Supersymmetric vacua and Bethe ansatz, arXiv:0901.4744 [INSPIRE].

[65] N.A. Nekrasov and S.L. Shatashvili, Quantum integrability and supersymmetric vacua, arXiv:0901.4748 [INSPIRE]. 
[66] T. Nishinaka, S. Sasa and R.-D. Zhu, On the correspondence between surface operators in Argyres-Douglas theories and modules of chiral algebra, JHEP 03 (2019) 091 [arXiv: 1811.11772] [INSPIRE].

[67] Y. Pan and W. Peelaers, Schur correlation functions on $S^{3} \times S^{1}$, JHEP 07 (2019) 013 [arXiv: 1903.03623] [INSPIRE].

[68] Y. Pan and W. Peelaers, Deformation quantizations from vertex operator algebras, arXiv:1911.09631 [INSPIRE].

[69] L. Rastelli, private communication.

[70] A Rocha-Caridi, Vacuum vector representations of the Virasoro algebra, in Vertex Operators in Mathematics and Physics, J. Lepowsky et al., eds., Springer, Germany (1985).

[71] L.J. Rogers, Second memoir on the expansion of certain infinite products, Proc. London Math. Soc. 25 (1894) 318.

[72] L.J. Rogers, On two theorems of combinatory analysis and some allied identities, Proc. London Math. Soc. 16 (1917) 315.

[73] L.J. Rogers and S. Ramanujan, Proof of certain identities in combinatory analysis, Proc. Cambridge Phyl. Soc. 19 (1919) 211.

[74] L.J. Slater, Further identities of the Rogers-Ramanujan type, Proc. London Math. Soc. 54 (1952) 147.

[75] J. Song, Superconformal indices of generalized Argyres-Douglas theories from $2 d$ TQFT, JHEP 02 (2016) 045 [arXiv: 1509.06730] [INSPIRE].

[76] J. Song, Macdonald index and chiral algebra, JHEP 08 (2017) 044 [arXiv:1612.08956] [INSPIRE].

[77] J. Song, D. Xie and W. Yan, Vertex operator algebras of Argyres-Douglas theories from M5-branes, JHEP 12 (2017) 123 [arXiv:1706.01607] [INSPIRE].

[78] D. Xie and W. Yan, $W$ algebra, cosets and VOAs for $4 d \mathcal{N}=2$ SCFT from M5 branes, arXiv: 1902.02838 [INSPIRE].

[79] D. Xie and W. Yan, Schur sector of Argyres-Douglas theory and W-algebra, arXiv:1904.09094 [INSPIRE].

[80] D. Xie and W. Yan, $4 d \mathcal{N}=2$ SCFTs and Lisse $W$-algebras, arXiv:1910.02281 [INSPIRE].

[81] S. Warnaar, Fermionic solution of the Andrews-Baxter-Forrester model: 1. Unification of TBA and CTM methods, hep-th/9501134 [INSPIRE].

[82] S.O. Warnaar, Fermionic solution of the Andrews-Baxter-Forrester model. 2: Proof of Melzer's polynomial identities, J. Statist. Phys. 84 (1996) 49 [hep-th/9508079] [InSPIRE].

[83] S.O. Warnaar, The Andrews-Gordon identities and q-multinomial coefficients, Commun. Math. Phys. 184 (1997) 203 [q-alg/9601012].

[84] S.O. Warnaar, Hall-Littlewood functions and the $A_{2}$ Rogers-Ramanujan identities, Adv. Math. 200 (2006) 403 [math.CO/0410592].

[85] A. Watanabe and R.-D. Zhu, Testing Macdonald index as a refined character of chiral algebra, JHEP 02 (2020) 004 [arXiv: 1909.04074] [INSPIRE].

[86] T.A. Welsh, Fermionic expressions for minimal model Virasoro characters, Mem. Amer. Math. Soc. 175 (2005) [math. Co/0212154]. 